\title{
Radiation-Enhancement Properties of an X-Band Woodpile EBG and Its Application to a Planar Antenna
}

\author{
Fabrizio Frezza, ${ }^{1}$ Lara Pajewski, ${ }^{2}$ Emanuele Piuzzi, ${ }^{1}$ \\ Cristina Ponti, ${ }^{2}$ and Giuseppe Schettini ${ }^{2}$ \\ ${ }^{1}$ Department of Information Engineering, Electronics and Telecommunications, "Sapienza" University of Rome, \\ Via Eudossiana 18, 00184 Roma, Italy \\ ${ }^{2}$ Department of Engineering, "Roma Tre" University of Rome, Via della Vasca Navale 84, 00146 Roma, Italy
}

Correspondence should be addressed to Lara Pajewski; lara.pajewski@uniroma3.it

Received 29 April 2013; Accepted 18 October 2013; Published 21 January 2014

Academic Editor: Tat Yeo

Copyright (C) 2014 Fabrizio Frezza et al. This is an open access article distributed under the Creative Commons Attribution License, which permits unrestricted use, distribution, and reproduction in any medium, provided the original work is properly cited.

\begin{abstract}
A woodpile Electromagnetic Bandgap (EBG) material has been designed, by using an in-house code that implements the Fourier Modal Method (FMM). A couple of alumina-woodpile samples have been fabricated. Several results have been collected for the transmission behaviour of the woodpile and of resonators with woodpile mirrors, in a shielded anechoic chamber, by using a vector network analyzer, in the $8-12 \mathrm{GHz}$ range. These new experimental data highlight interesting properties of 3D EBG resonators and suggest possible innovative applications. Comparisons of the collected results with FMM show a satisfactory agreement. An application of the EBG resonator has been considered, for gain enhancement of a microstrip antenna: an increase of about $10 \mathrm{~dB}$ in the broadside gain has been measured; experimental data and numerical results obtained with the commercial software HFSS show a good agreement. A comparison is presented between EBG resonator antennas and two-dimensional uniform arrays. Finally, HFSS results are provided for EBG resonator antennas working at higher frequencies or with a more selective superstrate: a gain enhancement of more than $18 \mathrm{~dB}$ is achieved by such antennas.
\end{abstract}

\section{Introduction}

Electromagnetic Bandgap (EBG) [1-3] structures, also called electromagnetic crystals, are subject to an increasing interest for their desirable properties that cannot be observed in natural materials. In this regard, they are classified as metamaterials [4].

The woodpile is an EBG that may present complete three-dimensional (3D) stop bands $[5,6]$. It is a stack of dielectric rods with alternating orthogonal orientations and its main advantage, compared to other geometries promoting the existence of complete bandgaps, is that it can be quite easily fabricated even for high-frequency applications. A woodpile can be realized as a sequence of layers, deposited and patterned by lithographic techniques developed for the semiconductor electronics industry [7], or by the extrusion freeforming technique [8]. Woodpile crystals with a complete band-gap centred at $2.35 \mu \mathrm{m}$ [9] and $1.55 \mu \mathrm{m}$ [10] have been realized through silicon double inversion of polymer templates and direct laser writing. This kind of EBG has been employed to realize waveguides [11, 12], waveguide bends [11$14]$, power dividers $[13,14]$, and as substrate [15] or superstrate [16] for the improvement of planar antenna performances.

The introduction of a defect in a crystal interrupts its periodicity and may cause the occurrence of a transmission peak in a stop band: for this reason, EBGs with defects are employed in the design of resonant cavities, filters, switches, and more $[17,18]$. A Fabry-Perot cavity with identical EBG mirrors can be considered as a crystal with a twin defect, that is, with a break of its periodicity obtained by a removal of one or more layers of elementary cells, such that one half of the resulting structure is a mirror image of the other one.

In [19], the reflection properties of a photonic crystal with square-section rods, with and without a planar defect, were studied numerically and experimentally; the presented reflection-phase measurements of photonic crystals were the first reported in the scientific literature. In 
[20], a Fabry-Perot resonator with one-dimensional photonic band-gap mirrors was analyzed: the authors focused on the case of a structure with a couple of two-layer periodic mirrors surrounding a homogeneous layer, explaining the influence of design parameters on the position and tunability of defect modes; experimental results, obtained through time-domain terahertz spectroscopy, were published in [21]. The transmission properties of cavities with twodimensional EBG mirrors were theoretically and numerically studied in [22], where the effects of the variation of physical and geometrical parameters were investigated and the application of the considered structures as filters was discussed. Experimental works at microwave frequencies on EBG cavities are not frequent, and in most of the published papers numerical results of simulations are reported.

An application of the Fabry-Perot cavity with EBG mirrors is the EBG resonator antenna. In this kind of antenna, an electromagnetic crystal is employed as a superstrate on a primary radiator, backed with a ground plane, and its main effect is a considerable increase in the directivity $[3,16,23-$ 25]. The EBG resonator antenna appears to be an alternative to aperture antennas, as parabolic antennas or lenses, or to antenna arrays, since it does not present some problems inherent to the usual directive systems, such as the size of the focal structures, or the limitations or complications induced by the feeding circuit of arrays. The superstrate of an EBG resonator antenna has to be placed at a distance equal to an integer multiple of half a wavelength from a source backed with a ground plane, or from a radiating aperture. The forward radiation is remarkably enhanced by means of in-phase multiple reflections. Various configurations were proposed in the literature, with a microstrip patch $[16,24]$, slots [16], a rectangular [8] or circular [25] waveguide feed, or else a conical horn [8], as primary radiator. Even if the single-feed case is the most studied, allowing the gain to be increased with low complexity, also arrays of microstrip patches or horns were considered as sources of EBG resonator antennas [8]. Systems operating at different frequencies, ranging from the microwave region to millimeter waves, were studied. EBGs with one, two, or three periodicity directions were used as superstrates.

Instead of using a ground-plane and an EBG superstrate, it is possible to put a source inside a Fabry-Perot cavity [26, 27]. Moreover, a Fabry-Perot cavity can be adopted as a cover instead of a single EBG superstrate [8, 28].

Sectoral horn antennas made of woodpile material have also been studied. In this kind of antennas, the electromagnetic confinement mechanism within the horn relies wholly on the $3 \mathrm{D}$ EBG, which is flared in one principal plane; the antenna shows a typical sectoral horn pattern, with a directional beam in one principal plane and a broader beam in the other [29]. Linear arrays of woodpile-EBG sectoral horn antennas have been studied in [30].

This work focuses on woodpile-EBG resonators and on woodpile-EBG resonator antennas. Several novel results of experiments and simulations are presented.

First of all, we resume the synthesis of a woodpile EBG with a complete band-gap centred on $f=12 \mathrm{GHz}$ and extending over almost $4 \mathrm{GHz}$ (Section 2). This design was performed by using an in-house code that implements the Fourier Modal Method (FMM) [31-34]. A couple of woodpile samples were fabricated, by means of alumina rods with square cross section, as briefly described in Section 3.

In a shielded anechoic chamber, in the $8-12 \mathrm{GHz}$ frequency range, we measured the transmission properties of our woodpile samples and of EBG Fabry-Perot cavities with different lengths and orientation of the mirrors. As already mentioned, experiments at microwave frequencies on EBG cavities have rarely been published, in most of the scientific papers only results of simulations are presented. We collected new interesting experimental results, which are reported and commented on in Sections 4.1 and 4.2. We carried out comparisons of experimental data with FMM results, achieving a good agreement. Moreover, we performed simulations of EBG cavities different from the measured prototypes: the obtained results are useful to highlight some interesting properties of cavities with $3 \mathrm{D}$ periodic mirrors and suggest possible innovative applications of this kind of structures.

Subsequently, we applied the EBG resonators to achieve gain enhancement of planar antennas; see Section 4.3. By putting a woodpile sample at various appropriate distances from a printed rectangular patch antenna, we obtained an enlargement of the equivalent-aperture area of the antenna, resulting in high-gain radiation. The return loss, the gain, and the radiation pattern in the $\mathrm{E}$ - and $\mathrm{H}$-planes were measured for the woodpile-covered microstrip antenna, changing the orientation of the EBG and its distance from the antenna. The increase in broadside gain was about $10 \mathrm{~dB}$. We performed comparisons with the commercial software Ansoft HFSS, with satisfactory agreement. We also performed a comparison with the gain-enhancement properties of twodimensional uniform arrays. Finally, by using HFSS we simulated a woodpile-covered antenna working at a higher frequency, showing that a higher gain can be obtained; furthermore, we considered a configuration with a thicker superstrate, constituted by two woodpile layers, also leading to a stronger gain enhancement of more than $18 \mathrm{~dB}$.

The conclusions of this work and ideas for future developments are drawn in Section 5.

\section{Design of a Woodpile EBG}

A woodpile was designed, by using an in-house code implementing the FMM, a full-wave approach that solves the monochromatic plane-wave scattering problem by crossed gratings. In the FMM, the simulated structure has a finite thickness in a periodicity direction and an infinite extension in the transverse plane; interruptions of periodicity can be treated; field polarization and incidence direction are arbitrary; therefore, off-plane stop-band structures can be calculated. In the FMM simulations presented in this section, the involved materials are supposed to be lossless.

In Figure 1 a scheme of the unit cell of a woodpile with square-section rods is given. The cross section of the woodpile inclusions may be rectangular or circular, or else 


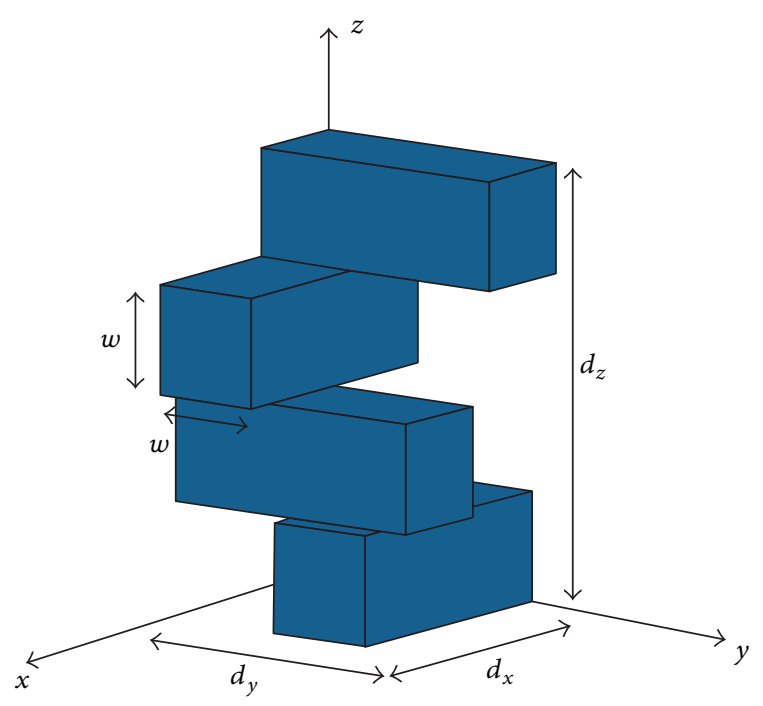

FIGURE 1: Scheme of the unit cell of a woodpile with square-section rods.

air cylindrical holes may be drilled in a dielectric sample; however, a woodpile with square-section rods is often preferred, because it is easier to fabricate. Rods belonging to two consecutive layers are orthogonal, and parallel rods are half a period offset.

Our initial goal was to design a woodpile with a gap centred on $12 \mathrm{GHz}$, possibly extending over a large frequency interval.

The design parameters of a woodpile unit cell with square-section rods are the dielectric relative permittivity $\varepsilon_{r}$, the side-length $w$ of the rod-section, and the periods $d_{x}, d_{y}$, and $d_{z}$ along the three orthogonal directions of periodicity. For a given geometry of the unit cell, there are typically no gaps until the filling factor and the dielectric contrast between rods and air are increased to some threshold values; above them, one or more gaps open up, with width usually increasing monotonically with the dielectric contrast. Moreover, a stop band becomes more pronounced as the number of woodpile layers stacked in the EBG is increased. We opted for alumina bars, to achieve a high dielectric contrast with low losses and strong stop bands with a compact structure.

We performed several simulations, calculating the field reflected and transmitted by different woodpile structures when a unit-amplitude monochromatic plane wave impinges from the negative $z$ half space (assuming that $z$ is the direction in which the crystal has a finite-thickness). The wavenumber of the incident field is $k=2 \pi / \lambda$, with $\lambda$ being the wavelength of the electromagnetic field in air. The wavevector is $\mathbf{k}=\alpha \widehat{\mathbf{x}}+\beta \widehat{\mathbf{y}}+\gamma \widehat{\mathbf{z}}$, with $\alpha=k \sin \theta \cos \varphi, \beta=$ $k \sin \theta \sin \varphi$, and $\gamma=k \cos \theta$. The electric-field polarization unit vector is $\widehat{\mathbf{u}}=(\cos \psi \cos \theta \cos \varphi-\sin \psi \sin \varphi) \widehat{\mathbf{x}}+$ $(\cos \psi \cos \theta \sin \varphi+\sin \psi \cos \varphi) \widehat{\mathbf{y}}-\cos \psi \sin \theta \widehat{\mathbf{z}}$. The incidence angle is denoted with $\theta, \varphi$ is the azimuthal angle, and $\psi$ is the angle between the incidence plane and $\widehat{\mathbf{u}}[32]$.
Depending on the periods, a woodpile may exhibit stop bands at different frequencies. We verified that if a larger complete stop band is needed, a woodpile with $d_{x}=d_{y}=d_{z}$ should be preferred. Thus, we focused on solutions with $d_{x}=$ $d_{y}=d$; however, for practical reasons, we chose $d_{z}=4 w$ (so that consecutive layers could be placed on each other). It is interesting to note that, with an identical spacing in the three periodicity directions, a face-centred cubic lattice would be produced [1]. Otherwise, with $d_{x}=d_{y} \neq d_{z}$, the lattice is face-centred tetragonal, which can be thought of as a facecentred cubic lattice that has been stretched or compressed in a periodicity direction.

We also observed that a simple woodpile-like stack, with no shift between parallel rods belonging to different layers (hence, easier to fabricate), does not produce significant bandgaps: they are smaller than in the woodpile case and not complete.

We subsequently considered in more detail the behaviour of structures built with commercially available rods of size: $w=1.80,3.18,6.35$, or $12.70 \mathrm{~mm}$. Since an EBG typically exhibits stop bands when its period is few tenths of a wavelength, we found, as expected, that the smallest and largest bars were not suitable to our purpose. The best results have been obtained with $w=3.18 \mathrm{~mm}$ and $d=8 \mathrm{~mm}$. For this geometry, in Figure 2 the transmission through the woodpile, $\eta_{T}$, is plotted as a function of frequency $f ; N$ represents the number of unit cells stacked along $z$ (the total number of rod layers in this periodicity direction is therefore $4 N$ ) and the relative dielectric constant of alumina is $\varepsilon_{r, \mathrm{al}}=$ 9.8. The plane wave normally impinges on the woodpile and the electric field is $x$-polarized, and thus both the electric and the magnetic fields are parallel to woodpile rods (for symmetry reasons and since the involved media are lossless, the same results are obtained when the polarization of the electric field is along the $y$-axis). A band gap centred on $f=12 \mathrm{GHz}$ is observed, extending over almost $4 \mathrm{GHz}$.

We also studied the off-plane behaviour of the woodpile. In Figures 3 and 4 the same as in Figure 2 is shown, when $N=$ 2 , for different incidence $(\theta)$, azimuthal $(\phi)$, and polarization $(\psi)$ angles. The stop band is slightly shifted with respect to the case of Figure 2; however, it reveals to be scarcely sensitive to variations of both incidence direction and field polarization.

\section{Prototype Fabrication}

Two identical woodpile samples were realized. The diameter tolerance of the CoorsTek AD-998 alumina rods that we employed is $\pm 3 \%$, their straightness is represented by a camber/length parameter $\leq 0.003$, and the maximum twist is $2^{\circ}$ per $30.48 \mathrm{~cm}$. To obtain a woodpile enough wide, containing at the same time size and cost, we chose a length of $20 \mathrm{~cm}$ for the alumina rods, corresponding to 25 periods; it is almost 7 wavelengths at $10 \mathrm{GHz}$.

The alignment of the rods was performed by means of a metallic structure, specifically designed, with equally spaced steel pins mounted on the four sides of an aluminum plate. Each layer of the woodpile was formed placing the rods through two opposite rows of pins, with a distance $d$ 


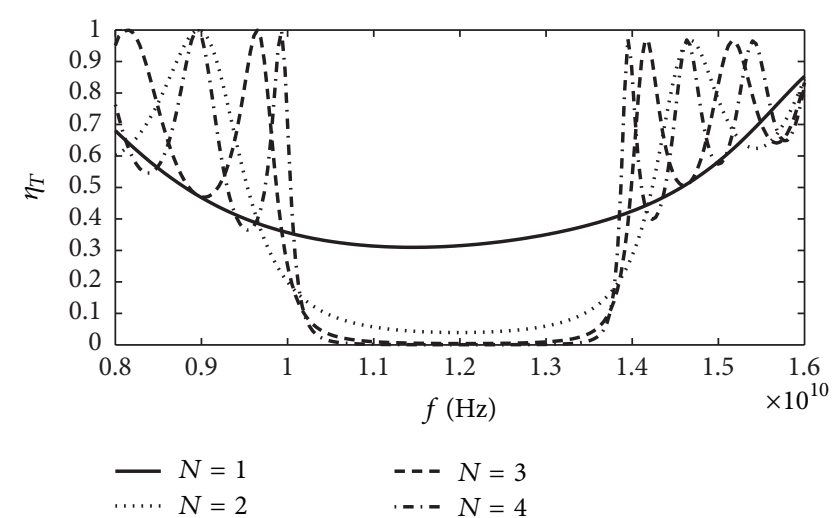

FIgURE 2: Transmission $\eta_{T}$ versus frequency when $w=3.18 \mathrm{~mm}$, $d=8 \mathrm{~mm}$, and $\varepsilon_{r, \mathrm{al}}=9.8$, for normal incidence and $x$-polarized electric field.

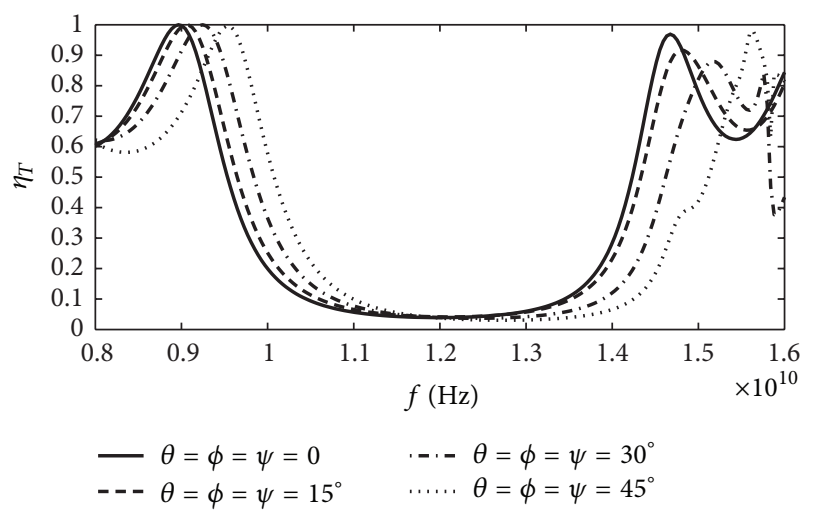

Figure 3: The same as in Figure 2, when $N=2$, for different incidence $(\theta)$, azimuthal $(\phi)$, and polarization $(\psi)$ angles.

between two consecutive rods. Once a layer was arranged, the neighbouring one was obtained by using the two orthogonal rows of pins, thus achieving a rotation of $90^{\circ}$ among adjacent rod gratings. Rods in contact were glued together. A picture of the resulting prototype is reported in Figure 5.

Moreover, we designed and realized a PVC table and a teflon/PVC support, to hold the woodpile samples during the experimental investigations. The scheme of the support and a picture of the woodpile prototypes on it are shown in Figure 6.

\section{Results}

In this Section, results of our measurements and simulations are presented.

All the experiments have been performed in a $3.20 \mathrm{~m} \times$ $3.20 \mathrm{~m} \times 2.70 \mathrm{~m}$ shielded anechoic chamber, using a HP 8530 vector network analyzer.

The first series of measurements concerned the transmission properties of both a single woodpile EBG and of woodpile cavities. The adopted setup consisted of a couple of $X$-band precision pyramidal horn antennas, placed one in front of the other, with the woodpile samples in the middle, held by the above described support.

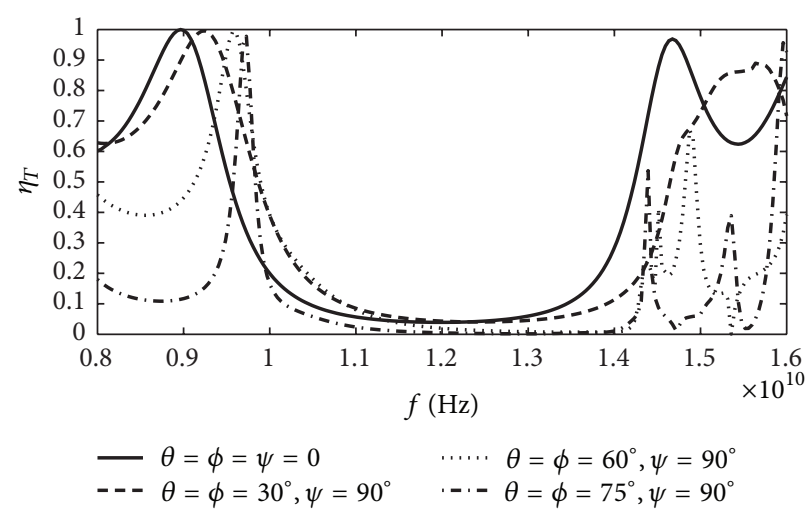

FIgURE 4: The same as in Figure 3, for some other incidence, azimuthal, and polarization angles.

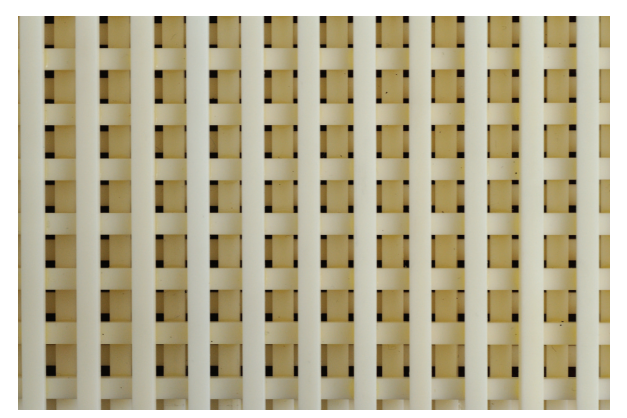

FIGURE 5: Picture of woodpile prototype.

In order to comply with the frequency band of the horn antennas, measurements were performed in the $8-12 \mathrm{GHz}$ range. The distance between the two antennas was chosen after a series of preliminary experiments aimed at ensuring a good compromise between the planarity of the wavefront impinging on the woodpile and the diffraction effects due to the finite extension of the samples.

The transmission properties of the woodpile samples were determined from the measured magnitude of the $S_{21}$ scattering parameter. To this aim, a preliminary reference calibration was performed with no sample placed between the two horns. Upon completion of each experimental session a further measurement was performed removing again the samples, in order to check that the drift in the measurement system was within acceptable levels.

The second series of measurements concerned the radiation properties of the EBG resonator antenna, constituted by a woodpile-covered patch. Measurements included return loss, gain, and radiation patterns in the $\mathrm{E}$ - and $\mathrm{H}$-planes. Only for the return loss, a different vector network analyzer was used: Agilent E8363B, preliminarily calibrated at the port by means of a SOL procedure and by employing the $85052 \mathrm{~B}$ mechanical calibration kit. Gain and radiation pattern measurements were performed with a setup similar to the one described for transmission properties characterization. The receiving horn antenna was replaced by the patch antenna, placed on a remotely controlled turntable. The distance between 


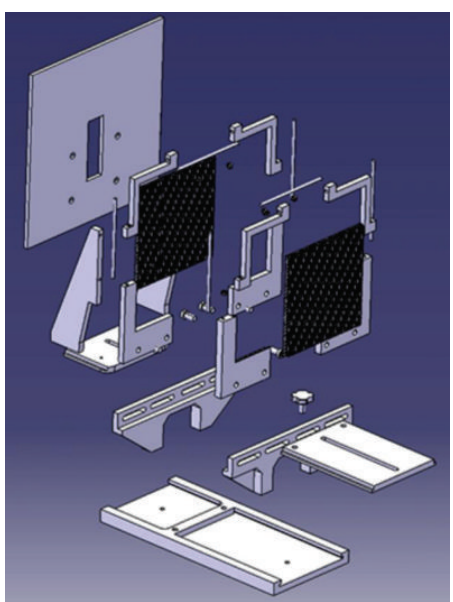

(a)

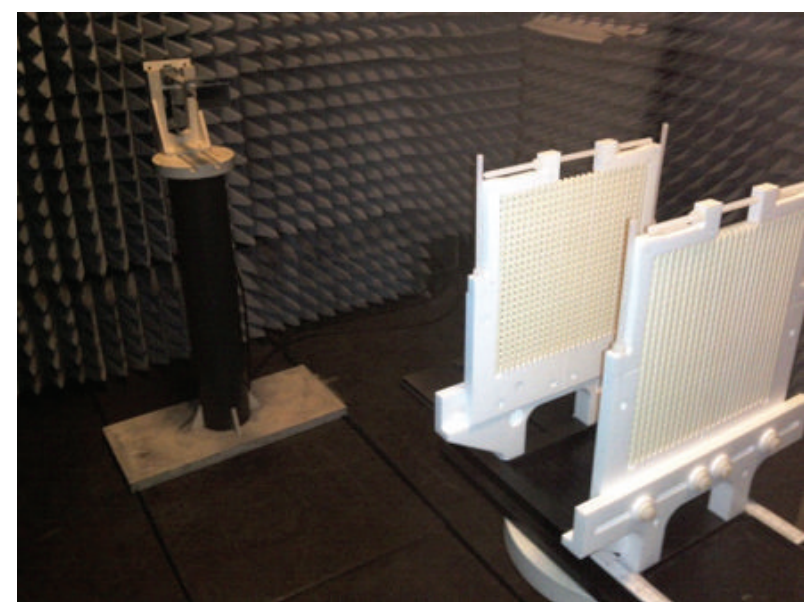

(b)

FIGURE 6: (a) Scheme of the teflon/PVC support. (b) The woodpile prototypes on the support.

the two antennas was chosen to be $1 \mathrm{~m}$, in order to be in the far field of the radiators. The setup was preliminarily subjected to a reference calibration with the receiving patch antenna replaced by the second standard gain horn antenna (broadside gain equal to $16 \mathrm{~dB}$ and standing-wave ratio equal to 1.25$)$.

4.1. Woodpile EBG. We characterized experimentally our woodpile, measuring its transmission properties.

In Figure 7, results for $\eta_{T}$ as a function of frequency are shown, obtained with the two prototypes juxtaposed and placed between the two precision horn antennas. It is worth citing that horn antennas have already been used in the past for the measurement of transmission through woodpile material; see Figures 3 and 4 in [16]. Our experimental curve in Figure 7 is compared with FMM results (the $N=2$ curve of Figure 2) and the agreement is good. Here and in the following, the differences between measured data and simulation results could be attributed to the fact that, in the FMM, the excitation is a monochromatic plane wave, the involved materials are supposed to be lossless, and above all the woodpile extension is infinite in the $x$ and $y$ directions; therefore, diffraction effects at the edges of the prototypes are neglected.

To give an idea of the electromagnetic effect of the PVC table and teflon/PVC support, $\eta_{T}$ has been measured when only horns were present (empty chamber) and when the table and the support, without woodpile prototypes, were positioned between the antennas: results as a function of frequency are reported in Figure 8. Moreover, in the same figure results for the transmission beyond a $20 \times 20 \mathrm{~cm}^{2}$ copper plane (held on the support, placed on the table) are shown. Analysis of Figure 8 shows that the effect of the table and support is rather negligible (as compared to the woodpile effect), with variations within $\pm 1 \mathrm{~dB}$. However, the curve recorded with the copper plane (having roughly the same transversal dimensions of the woodpile samples) shows that

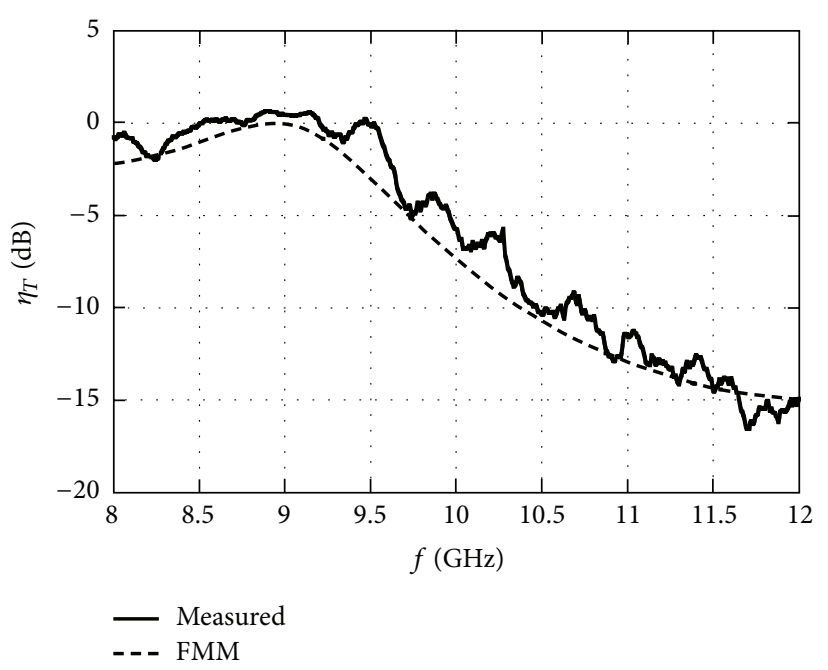

FIGURE 7: Transmission properties of the woodpile: $\eta_{T}$ as a function of frequency, $N=2$, measured data, and FMM results.

the effect of edge diffraction is clearly visible; nonetheless, its impact on the measured transmission curves with the EBG samples should not be so important, because of the limited shielding effectiveness of the samples themselves in the stop band (transmission generally above $-15 \mathrm{~dB}$, as shown in the following results).

4.2. Woodpile Cavity. We subsequently studied the electromagnetic behaviour of an EBG cavity, constituted by our woodpile prototypes placed at a distance $h$ from each other, as sketched in Figure 9. This is a symmetrical structure with respect to the $x y$ plane and it resonates when its equivalent length $L_{\text {eq }}$ is an integer multiple of $\lambda / 2$.

We placed the cavity between the horn antennas. This structure may also be considered as a woodpile with a break 

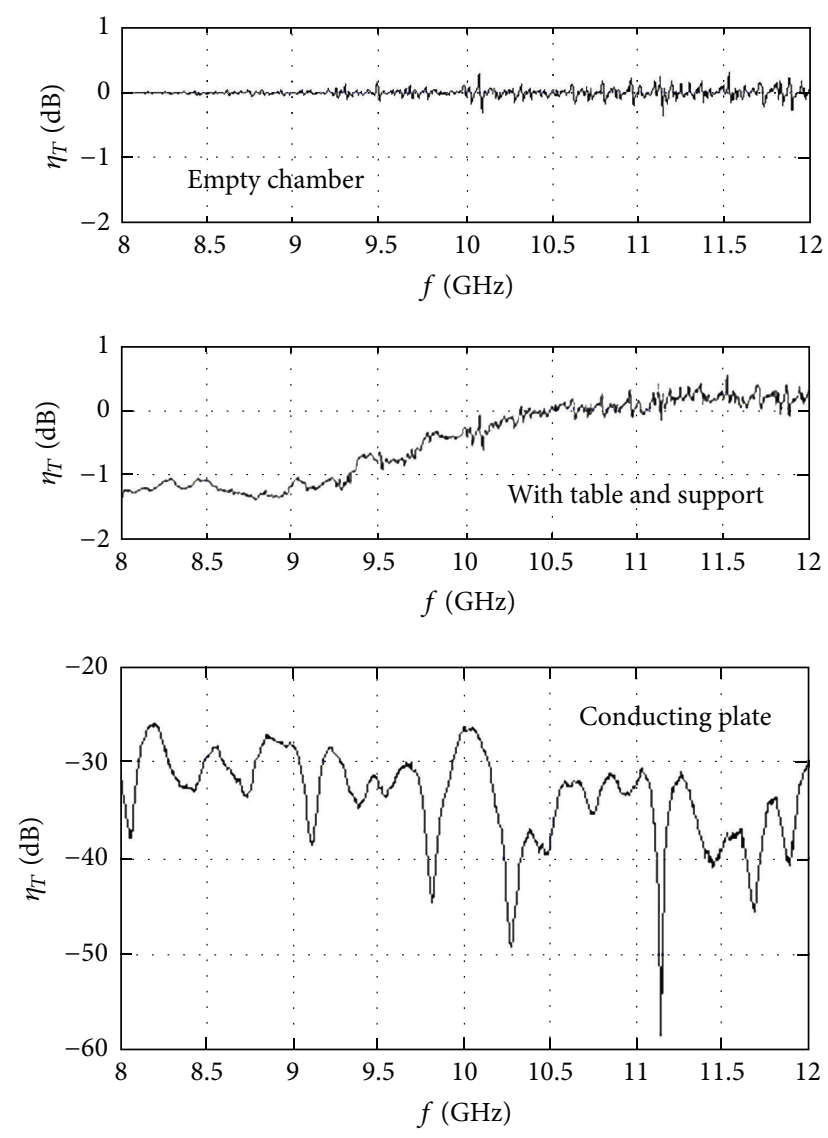

FIGURE 8: $\eta_{T}$ versus frequency for the empty anechoic chamber; in the presence of the table and the support; in the presence of a $20 \times$ $20 \mathrm{~cm}^{2}$ conducting plate held on the support, placed on the table.

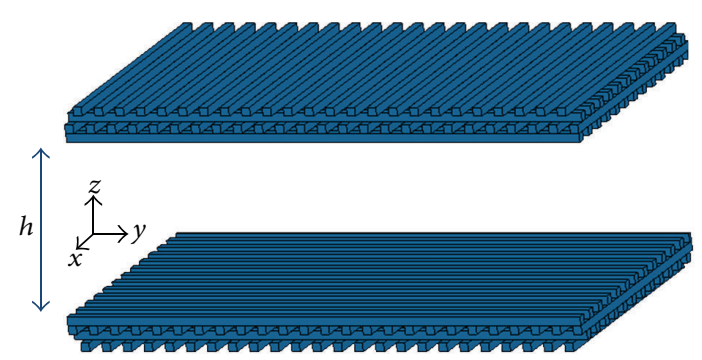

FIGURE 9: Sketch of a woodpile symmetrical cavity.

of its periodicity, in which some periodic layers of rods are replaced by a homogeneous air region with thickness $h$ in the $z$ direction.

In Figure 10, the transmission $\eta_{T}$ is reported, as a function of frequency, when $h=60,90$, and $119 \mathrm{~mm}$ and the inner rods of the cavity are parallel to the electric field. The figure shows that a single periodicity interruption may cause several localized states within a stop band: in fact, each curve presents various transmission peaks. In longer cavities, a higher number of resonances occurs within the same frequency range, as expected. Moreover, all the structures considered in the figure resonate at $10.3 \mathrm{GHz}$, and it is noted

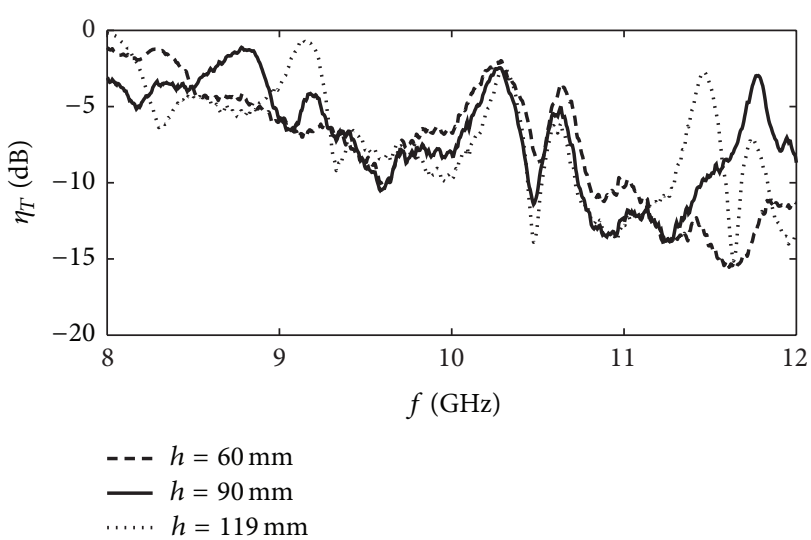

FIGURE 10: $\eta_{T}$ versus frequency (measured results): $h=60,90$, and $119 \mathrm{~mm}$, and the electric field is parallel to the inner rods of the cavity.

that the relevant peak has a higher quality factor when the cavity is longer.

The experimental curve for $h=90 \mathrm{~mm}$ was presented in [35], where it was compared, with good agreement, with FMM results. Let us now consider more in depth just this case. For such a cavity, three transmission peaks are observed. They are centred on $f_{1} \cong 8.80 \mathrm{GHz}, f_{2} \cong 10.30 \mathrm{GHz}$, and $f_{3} \cong$ $11.76 \mathrm{GHz}$. At these frequencies, the wavelength in air turns out to be $\lambda_{1} \cong 34.1 \mathrm{~mm}, \lambda_{2} \cong 29.1 \mathrm{~mm}$, and $\lambda_{3} \cong 25.5 \mathrm{~mm}$, respectively. Then, it can be deduced that $L_{\mathrm{eq}} \cong 102 \mathrm{~mm}$ $\cong 6 \lambda_{1} / 2 \cong 7 \lambda_{2} / 2 \cong 8 \lambda_{3} / 2$ : this means that the equivalent length of the cavity covers the whole air region plus about half thickness of both the EBG mirrors $\left(L_{\mathrm{eq}} \cong h+4 w\right)$. This result is very interesting, and the same is found if the $h=60 \mathrm{~mm}$ and $h=119 \mathrm{~mm}$ cases are examined.

The equivalent length of the cavity is highly dependent on the electromagnetic field polarization with respect to the woodpile orientation. We also measured cavities having the inner rods orthogonal to the electric field. An example is given in Figure 11. We verified that a resonance at $10.3 \mathrm{GHz}$, as in Figure 10, is obtained, when $h=26,84$, and $142 \mathrm{~mm}$. Analyzing the experimental results plotted in Figure 11, it turns out that when the electric field is orthogonal to the inner rods of the cavity, the equivalent length covers the whole air region plus the thickness of three periodic layers of both the mirrors $\left(L_{\mathrm{eq}} \cong h+6 w\right)$.

As a consequence, it seems that the periodic arrangement of bars perpendicular to the electric field has a negligible effect on the transmission through the whole structure. This deduction is confirmed by the FMM results shown in Figure 12, where $\eta_{T}$ is plotted, as a function of frequency, when $h=90 \mathrm{~mm}$. The behaviour of three different structures is compared in this figure: the solid line refers to a twodimensional EBG cavity obtained from the woodpile cavity relevant to Figure 10, by removing the bars orthogonal to the electric field (consequently, only bars parallel to the electric field are present); on the contrary, the dotted line refers to a cavity obtained by removing the bars parallel to the electric field (so, the remaining bars are perpendicular to the electric 


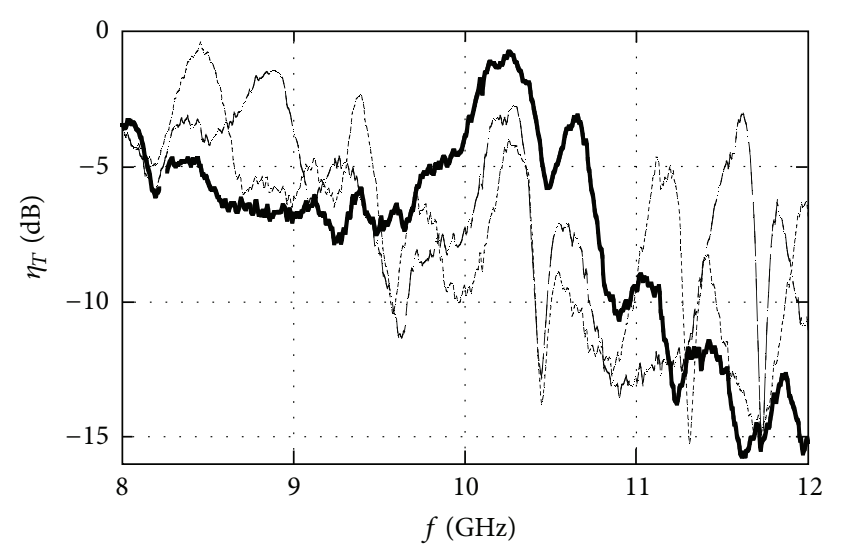

$$
\begin{aligned}
-h & =26 \mathrm{~mm} \\
---h & =84 \mathrm{~mm} \\
\ldots \ldots h & =142 \mathrm{~mm}
\end{aligned}
$$

FIGURE 11: $\eta_{T}$ versus frequency (measured results); $h=26,84$, and $142 \mathrm{~mm}$, and the electric field is orthogonal to the inner rods of the cavity.
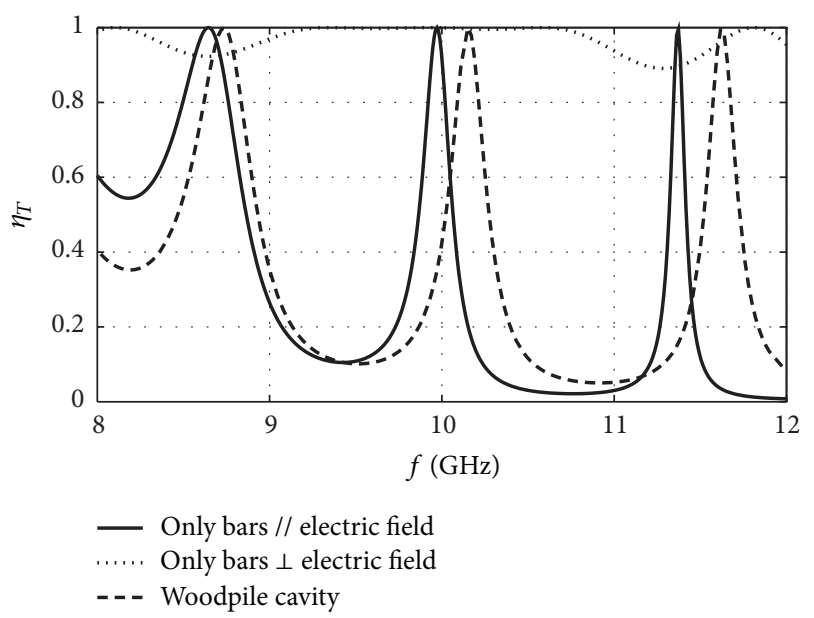

FIGURE 12: $\eta_{T}$ versus frequency (FMM results), when $h=90 \mathrm{~mm}$ and only rods parallel (solid line) or orthogonal (dotted line) to the electric field are present, or else the whole woodpile cavity is considered (dashed line).

field); the dashed line corresponds to the reference case of woodpile cavity and it has been reported for comparison. It can be appreciated that when only rods perpendicular to the electric field are present, the transmission through the structure is almost unitary; when only rods parallel to the electric field are present, the peaks are slightly shifted with respect to the woodpile case. These results suggest that adjacent layers of a woodpile might be independently designed, to interact with different linear sources having the electric field parallel to their rods, or to modify and control the polarization of an elliptically polarized field.

Finally, in Figure 13, results are reported for $h=99 \mathrm{~mm}$ and inner rods of the cavity orthogonal to the electric field. Two different curves are plotted, obtained when the electric field illuminating the cavity was perpendicular to the

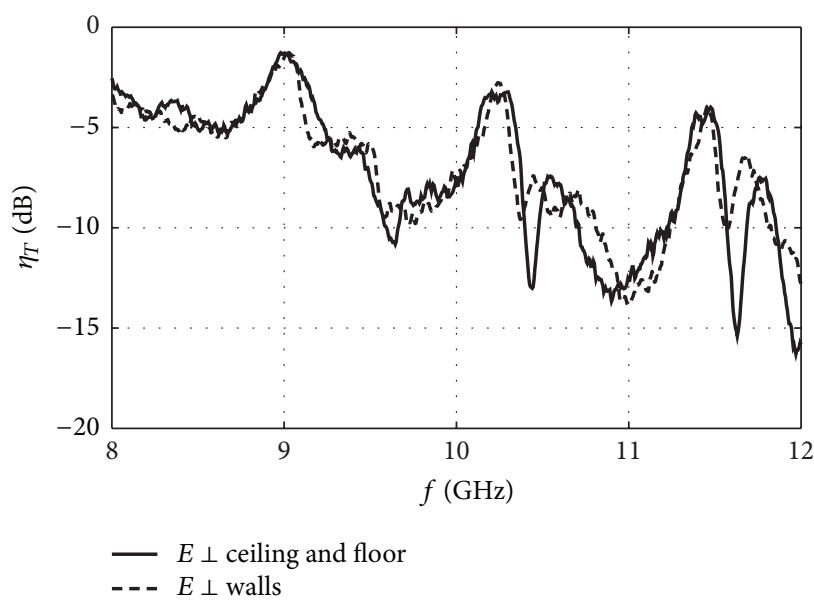

FIGURE 13: $\eta_{T}$ versus frequency (measured results); $h=99 \mathrm{~mm}$ and the electric field is orthogonal to the inner rods of the cavity. The reported results have been obtained with the electric field orthogonal to the ceiling and the floor (full line), or to the side walls (dotted line), of the anechoic chamber.

ceiling and the floor, or to the side walls, of the anechoic chamber. This figure gives an idea of the anechoicity of our environment and of the reliability of our measurements. The frequency behaviour of $\eta_{T}$ is qualitatively the same for both curves. It is important to notice that the resonances are coincident: this means that they are revealed with good accuracy in our experimental setup.

4.3. Gain Enhancement of a Microstrip Antenna. An interesting and promising application of EBG resonators is their use for antenna-gain enhancement, as discussed in Section 1.

According to the image theory, a configuration equivalent to the cavity of Figure 9 is obtained, if it is halved with respect to its symmetry plane with a perfectly conducting surface. If the ground plane of a patch antenna is employed as the perfectly conducting surface, the compound radiator sketched in Figure 14 is obtained.

In previous works, we already simulated radiators made of a microstrip antenna with a woodpile superstrate [33, 34]. We presented preliminary experimental results for a woodpile-covered microstrip antenna in [35]. In this subsection, we report and comment on several new results of both experiments and simulations, concerning a woodpilecovered microstrip rectangular patch. In [16], a structure similar to the one considered here is studied: it is a microstrip rectangular patch with a woodpile superstrate and few measured results are given, at $12.47 \mathrm{GHz}$, for the return loss, the gain, and the radiation patterns in $\mathrm{E}$ - and $\mathrm{H}$-planes.

The microstrip antenna used in this work was realized from a $0.76 \mathrm{~mm}$ thick Rogers/RT Duroid 5870 layer (relative permittivity 2.33), printed on both sides with $36 \mu \mathrm{m}$ thick copper. With a PC-controlled milling table, on one side of the layer a $8 \mathrm{~mm} \times 8.4 \mathrm{~mm}$ rectangular patch was cut. The antenna is fed from below by a coaxial probe (SMA connector): the feed point, where the probe is attached to the patch, is centred with respect to the shorter side of the patch 


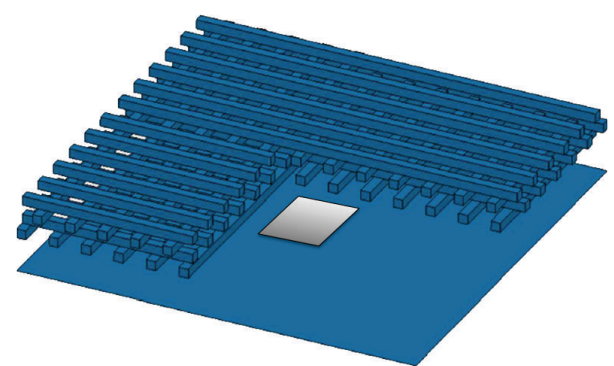

FIGURE 14: Sketch of the patch antenna with the woodpile superstrate.

and it is $1.2 \mathrm{~mm}$ away from the centre of the longer side. This antenna has been designed to work in $X$-band, to comply with the operating band of our precision horn antennas. Measurements of the return loss of the patch antenna revealed that it resonates at $10.3 \mathrm{GHz}$. At this frequency, the magnitude of the return loss is $-11.69 \mathrm{~dB}$ and the broadside gain is $6.18 \mathrm{~dB}$.

We characterized our woodpile-covered patch antenna by positioning the superstrate at various distances from the antenna, corresponding to half length $(h / 2)$ of cavities resonating at $10.3 \mathrm{GHz}$. At such distances, we first studied how the EBG affects the antenna matching. In Figure 15, the magnitude of the measured scattering parameter $S_{11}$ is plotted, as a function of frequency, for the patch antenna alone. In Figure 16, the same as in Figure 15 is reported for the woodpile-covered patch, for different values of $h$; the bars nearest to the patch are parallel to the electric field. It can be appreciated that the presence of the EBG superstrate does not significantly change the frequency behaviour of the return loss.

We verified that achieving a good parallelism between the ground-plane and the woodpile is very important to maximize the performances of the compound antenna. Moreover, we observed that a woodpile shift, parallel to the ground plane, does not affect the behaviour of the radiator. The orientation of the superstrate, instead, is very important, as in woodpile cavities.

In Figure 17, the broadside gain of the woodpile-covered patch antenna, $G_{\mathrm{pw}}$, normalized to the broadside gain of the patch alone, $G_{p}$, is plotted as a function of frequency for the same woodpile orientation and $h$ values studied in Figure 16. The broadside gain enhancement due to the woodpile occurs at $10.3 \mathrm{GHz}$, when the structure is matched and the equivalent distance between the woodpile and the patch is roughly equal to an integer multiple of $\lambda / 2$ : multiple reflections are present between the antenna and the superstrate, and they are summed in phase at the interface between air and woodpile.

In Figure $18, G_{\mathrm{pw}}$ normalized to $G_{p}$ is reported, as a function of frequency, for the $h=90 \mathrm{~mm}$ case; the experimental curve is compared with results obtained by using the commercial software Ansoft HFSS, implementing the finite element method, and the agreement is quite satisfactory. The experimental results show that a gain enhancement of $10.21 \mathrm{~dB}$ occurs at $10.3 \mathrm{GHz}$, due to the EBG; the calculated

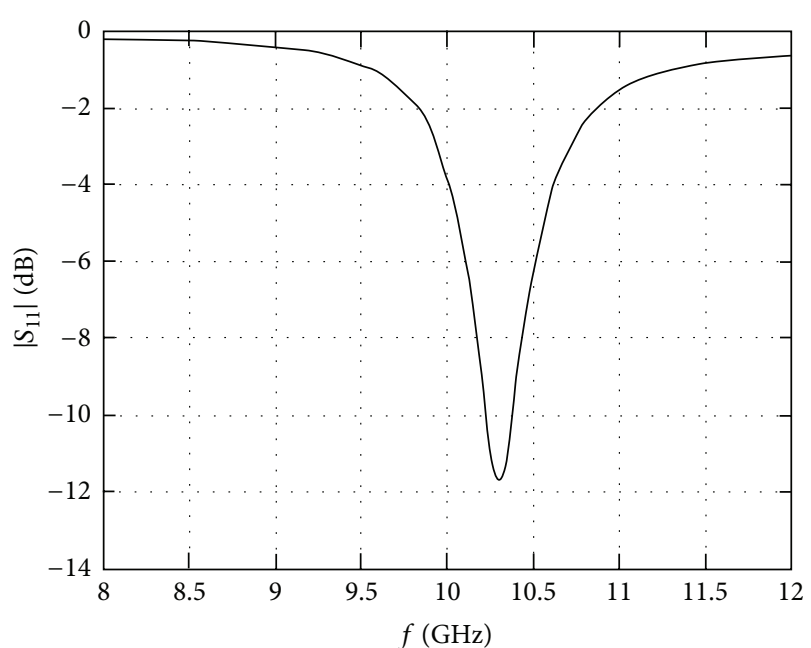

FIGURE 15: Return loss versus frequency for the rectangular microstrip antenna.

TABLE 1: Measured gain of the patch alone and of the woodpilecovered patch, in the $8-12 \mathrm{GHz}$ range $(h / 2=45 \mathrm{~mm})$.

\begin{tabular}{lcc}
\hline$f(\mathrm{GHz})$ & $G_{p}(\mathrm{~dB})$ & $G_{\mathrm{pw}}(\mathrm{dB})$ \\
\hline 8 & -3.88 & -5.96 \\
8.5 & 0.09 & -9.49 \\
9 & 1.47 & -4.11 \\
9.5 & 1.85 & -0.29 \\
10 & 4.61 & 7.02 \\
$\mathbf{1 0 . 3}$ & $\mathbf{6 . 1 8}$ & $\mathbf{1 6 . 3 9}$ \\
10.5 & 5.43 & 5.42 \\
11 & 1.52 & 4.39 \\
11.5 & 1.68 & -16.72 \\
12 & 1.24 & -4.63 \\
\hline
\end{tabular}

Results for the resonance frequency of the antenna, that is, $10.3 \mathrm{GHz}$, are reported in bold.

gain enhancement turns out to be slightly higher; it is $11.06 \mathrm{~dB}$. In Table 1 , the measured values of both $G_{p}$ and $G_{\mathrm{pw}}$ can be found, for different frequencies.

In Figure 19, the measured E- and H-plane radiation patterns $(\mathrm{dB})$ of the woodpile-covered patch antenna, normalized to their maximum values, are presented for the same case considered in Figure 18. Results for the patch alone are also reported, for comparison. The directivity enhancement due to the woodpile is strongly evident in the radiation patterns of Figure 19. For the EBG-covered antenna, the measured Half-Power Beamwidth (HPBW) is $12^{\circ}$ in the Eplane and $14^{\circ}$ in the H-plane; the Side-Lobe Level (SLL) is $-9.84 \mathrm{~dB}$ in the E-plane and $-13.15 \mathrm{~dB}$ in the H-plane. The experimental curves of Figure 19 are compared with the results obtained by using the HFSS software: it can be noted that the agreement is good and that the SLL predicted by HFSS is lower than the measured one in both the E- and Hplanes.

In Figure 20, the measured $\mathrm{H}$-plane radiation pattern is plotted for two different values of the distance between 

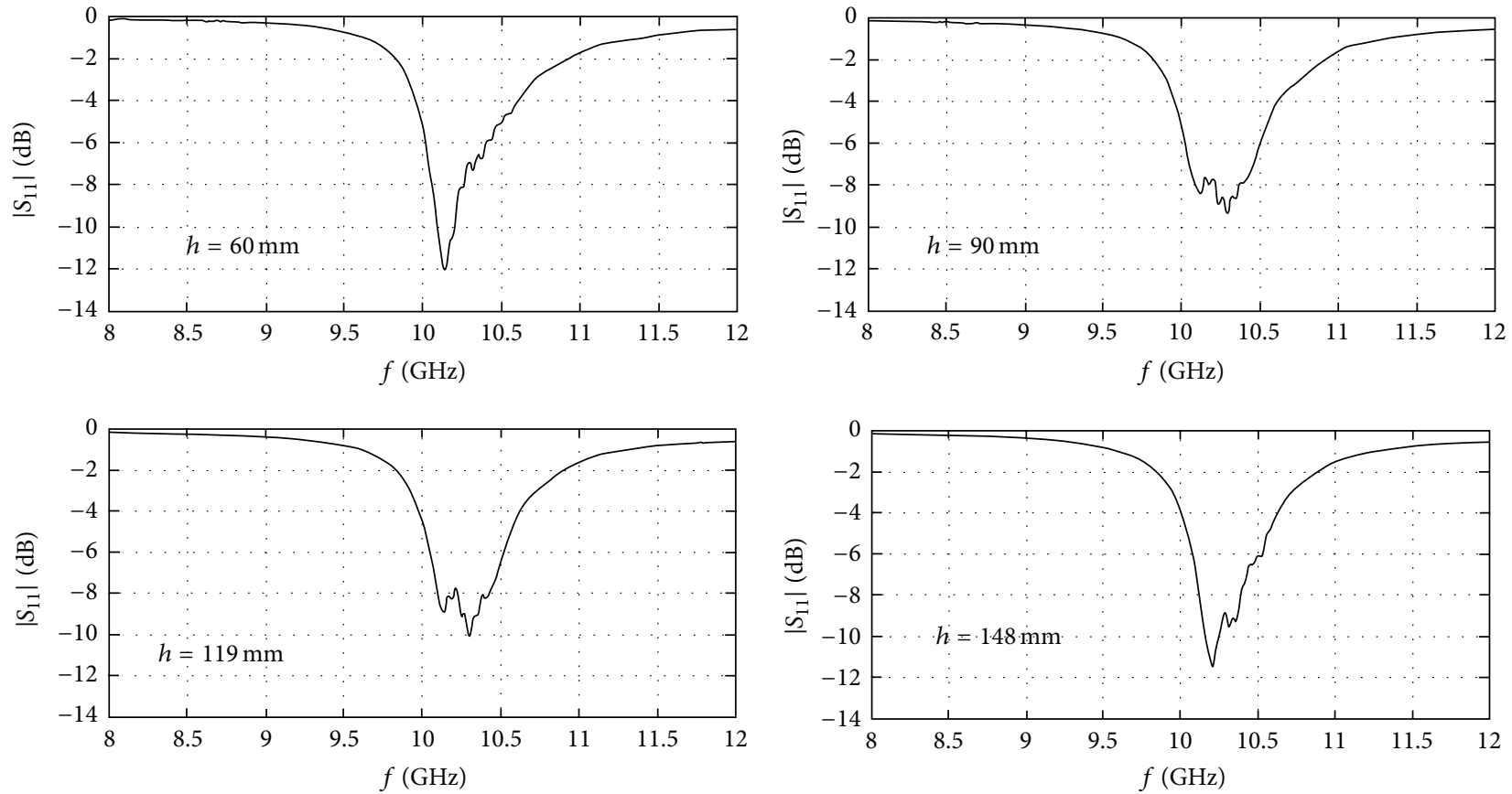

FIGURE 16: Return loss of the woodpile-covered patch antenna, versus frequency. Four different distances $(h / 2)$ between patch and woodpile are considered. The woodpile rods nearest to the ground plane are parallel to the electric field radiated by the patch.
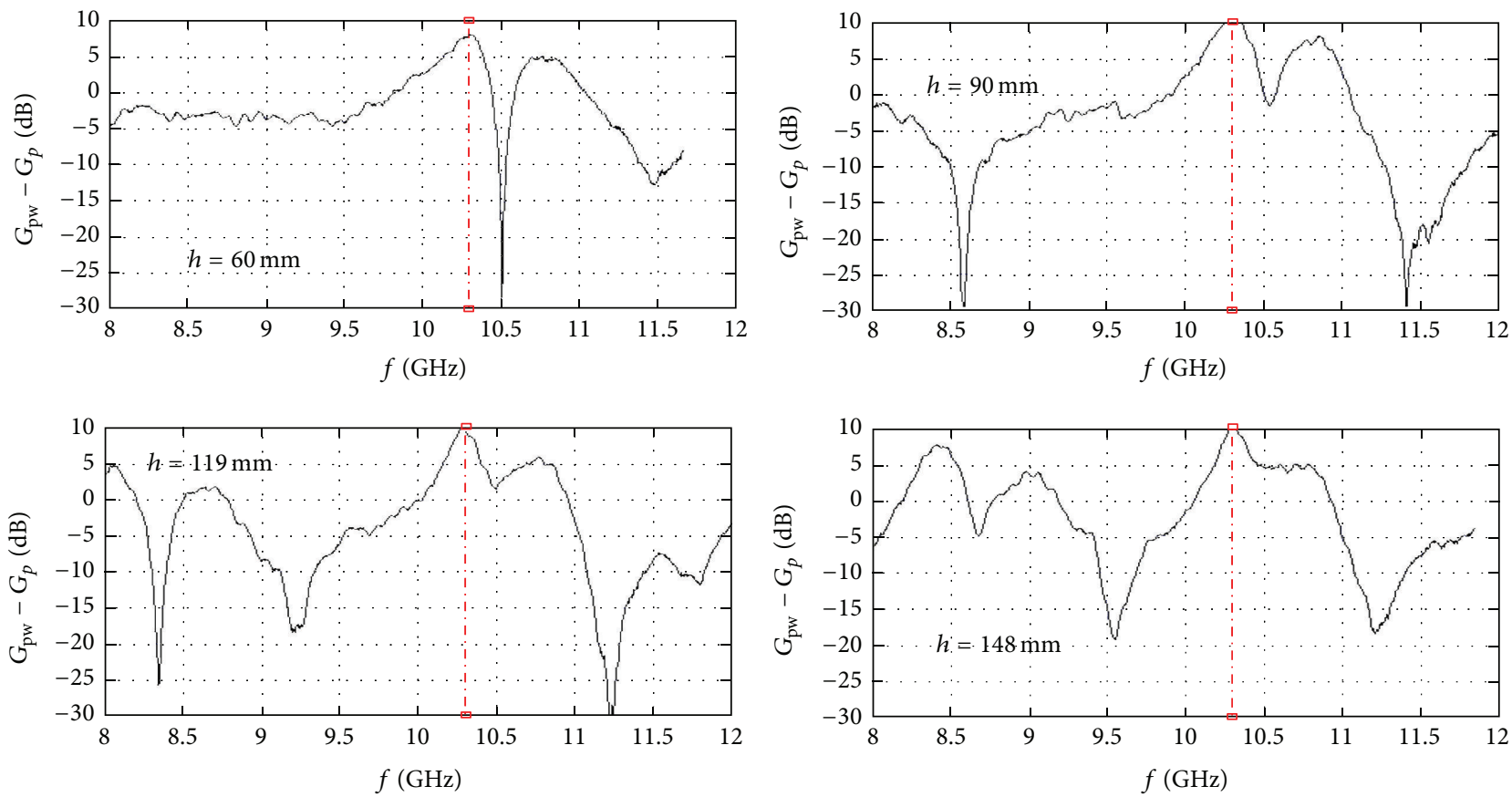

FIGURE 17: Broadside gain of the woodpile-covered patch antenna, $G_{\mathrm{pw}}$, normalized to the broadside gain of the patch alone, $G_{p}$, in $\mathrm{dB}$, versus frequency; $h$ assumes different values, and the woodpile bars nearest to the ground plane are parallel to the electric field radiated by the patch.

the EBG and the microstrip antenna: in particular, the case $h / 2=45 \mathrm{~mm}$, already shown in Figure 19, is compared with the case $h / 2=74 \mathrm{~mm}$. In both configurations the woodpile bars nearest to the patch are parallel to the electric field. It can be appreciated that, when the distance between patch and woodpile is larger, the main beam is narrower and the side-lobe level is higher. This is coherent with the results of Figure 10, where larger cavities show narrower resonance peaks.

It is interesting to perform a comparison between the gain enhancement obtained with the woodpile superstrate and the one that could be achieved with a two-dimensional 


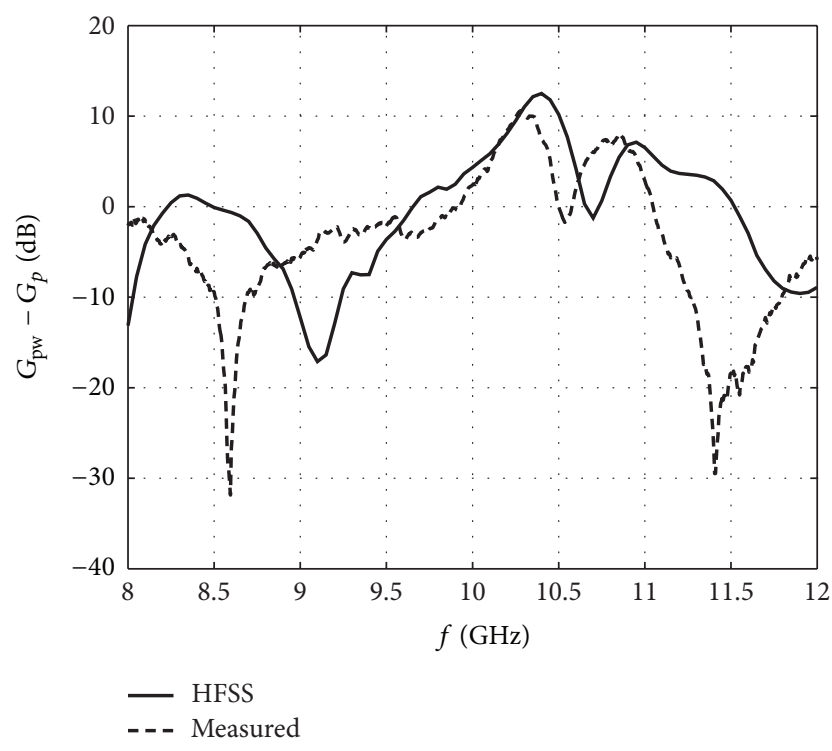

FIGURE 18: $G_{\mathrm{pw}}$ normalized to $G_{p}$, in $\mathrm{dB}$, versus frequency; the distance between patch and EBG is $h / 2=45 \mathrm{~mm}$ and the woodpile bars nearest to the patch are parallel to the electric field.

TABLE 2: Gain enhancement obtained by using a two-dimensional uniform array with $N_{a}$ elements.

\begin{tabular}{lcccc}
\hline$N_{a}$ & Max spacing & Opt spacing & Area $\left(\mathrm{cm}^{2}\right)$ & $\Delta G(\mathrm{~dB})$ \\
\hline 4 & $6.87 \lambda$ & $0.640 \lambda$ & $1.86 \times 1.86$ & 8.90 \\
9 & $3.43 \lambda$ & $0.740 \lambda$ & $4.31 \times 4.31$ & 13.56 \\
25 & $1.72 \lambda$ & $0.900 \lambda$ & $10.5 \times 10.5$ & 19.00 \\
49 & $1.14 \lambda$ & $0.880 \lambda$ & $15.4 \times 15.4$ & 22.30 \\
81 & $0.858 \lambda$ & $0.858 \lambda$ & $20.0 \times 20.0$ & 23.98 \\
\hline
\end{tabular}

array of patches occupying the same geometrical area as the woodpile. In Table 2, we report numerical results calculated for a uniform two-dimensional array with $N_{a}$ elements. The maximum area that the array can cover is a square equal to the woodpile extension: $20 \times 20 \mathrm{~cm}^{2}$. Consequently, once $N_{a}$ is fixed, the array spacing cannot be larger than $20 /\left(\sqrt{N_{a}}-1\right) \mathrm{cm}$ (second column). If the spacing specified in the third column of the table is chosen (giving the best results for the array with $N_{a}$ elements), at $10.3 \mathrm{GHz}$ the area of the array turns out to be the one given in the fourth column; the corresponding gain enhancement is reported in the fifth column of the table.

It is observed that, with a 9-element array, covering a fraction of the available area, a gain enhancement equal to $13.56 \mathrm{~dB}$ can be obtained. This value is slightly higher than the one expected for the woodpile-covered patch (we recall that the gain enhancement calculated by using HFSS is $11.06 \mathrm{~dB}$ when $h=90 \mathrm{~mm}$ ). If an array with a higher number of elements is considered, a stronger gain enhancement can be achieved; when 81 elements are present, the whole woodpile area is occupied and the gain enhancement is about $24 \mathrm{~dB}$.

These results show that, when a directive beam is needed, a woodpile cover can be considered a good alternative to an array not in the sense that it gives a higher increase of the gain. One of the benefits of the EBG resonator antenna resides in the possibility of obtaining a high gain with a device that uses a single feed, without the complications and limitations of a multiple-source radiating system. Moreover, as previously discussed, the matching of the antenna is slightly affected by the introduction of the periodic cover, while an array has a highly resonant matching.

We now present new results of FMM and HFSS simulations, suggesting that if the woodpile is used to cover a patch that resonates at higher frequencies, a stronger gain enhancement is obtained. Another possibility that we investigate, aiming at improving the gain enhancement, is the introduction of a superstrate with a higher number of periodic layers.

In Figure 21, FMM results are reported for a Fabry-Perot cavity with woodpile mirrors at a distance $h=90 \mathrm{~mm}$. The mirrors are constituted by one or two woodpile layers. The transmission through the cavity is plotted as a function of frequency in the $8-16 \mathrm{GHz}$ range. It is observed that with two woodpile layers the transmission peaks are sharper and slightly shifted toward higher or lower frequencies (they can be easily retuned to the desired frequency by changing the cavity length). In particular, if the peak near $10 \mathrm{GHz}$ is considered, it has a quality factor $Q=63.5$ with a single woodpile layer and $Q=157$ in the double-woodpile case. If the peak near $11 \mathrm{GHz}$ is examined, $Q=89.5$ and 687 with one and two woodpile layers, respectively. For the peak near $13 \mathrm{GHz}$, the quality factor is $Q=93.9$ and 938 with one and two woodpile layers, respectively. A higher quality factor of the cavity should result in a stronger gain enhancement of the corresponding EBG resonator antenna. Of course, with a double woodpile superstrate higher losses are present (we recall that in FMM simulations the involved materials are assumed to be lossless) partially cancelling the expected gain improvement.

The HFSS results reported in Figures 22-24 concern a woodpile-covered microstrip antenna working at $13.3 \mathrm{GHz}$. The patch is rectangular and its size is $6.2 \mathrm{~mm} \times 6.3 \mathrm{~mm}$. At 13.3 $\mathrm{GHz}$, with the woodpile at a distance $h / 2=45 \mathrm{~mm}$, the gain enhancement due to the presence of the EBG superstrate turns out to be $14 \mathrm{~dB}$ and the return loss of the compound radiator is less than $-20 \mathrm{~dB}$. In Figure 22, the return loss of the woodpile-covered antenna is plotted as a function of frequency and it is compared with the return loss of the patch alone: it can be noticed that the introduction of the superstrate has a negligible effect on the antenna matching; in particular the peak turns out to be slightly shifted toward lower frequencies. In Figure 23, the broadside gain of the woodpile-covered patch antenna, $G_{\mathrm{pw}}$, normalized to the broadside gain of the patch alone, $G_{p}$, in $\mathrm{dB}$, is plotted as a function of frequency. Furthermore, in Figure 24 we present the $\mathrm{E}$ - and $\mathrm{H}$-plane radiation patterns for this antenna; the $\mathrm{HPBW}$ is $8^{\circ}$ in the E-plane and $7^{\circ}$ in the H-plane; the SLL is $-12.78 \mathrm{~dB}$ in the E-plane and $-15.55 \mathrm{~dB}$ in the $\mathrm{H}$-plane. As expected, working at a higher frequency we obtained a stronger gain enhancement and more directive radiation patterns than at $10.3 \mathrm{GHz}$.

The HFSS results reported in Figures 25-27 concern an EBG resonator antenna working at $13.24 \mathrm{GHz}$, made of a microstrip patch covered by a superstrate constituted by two 

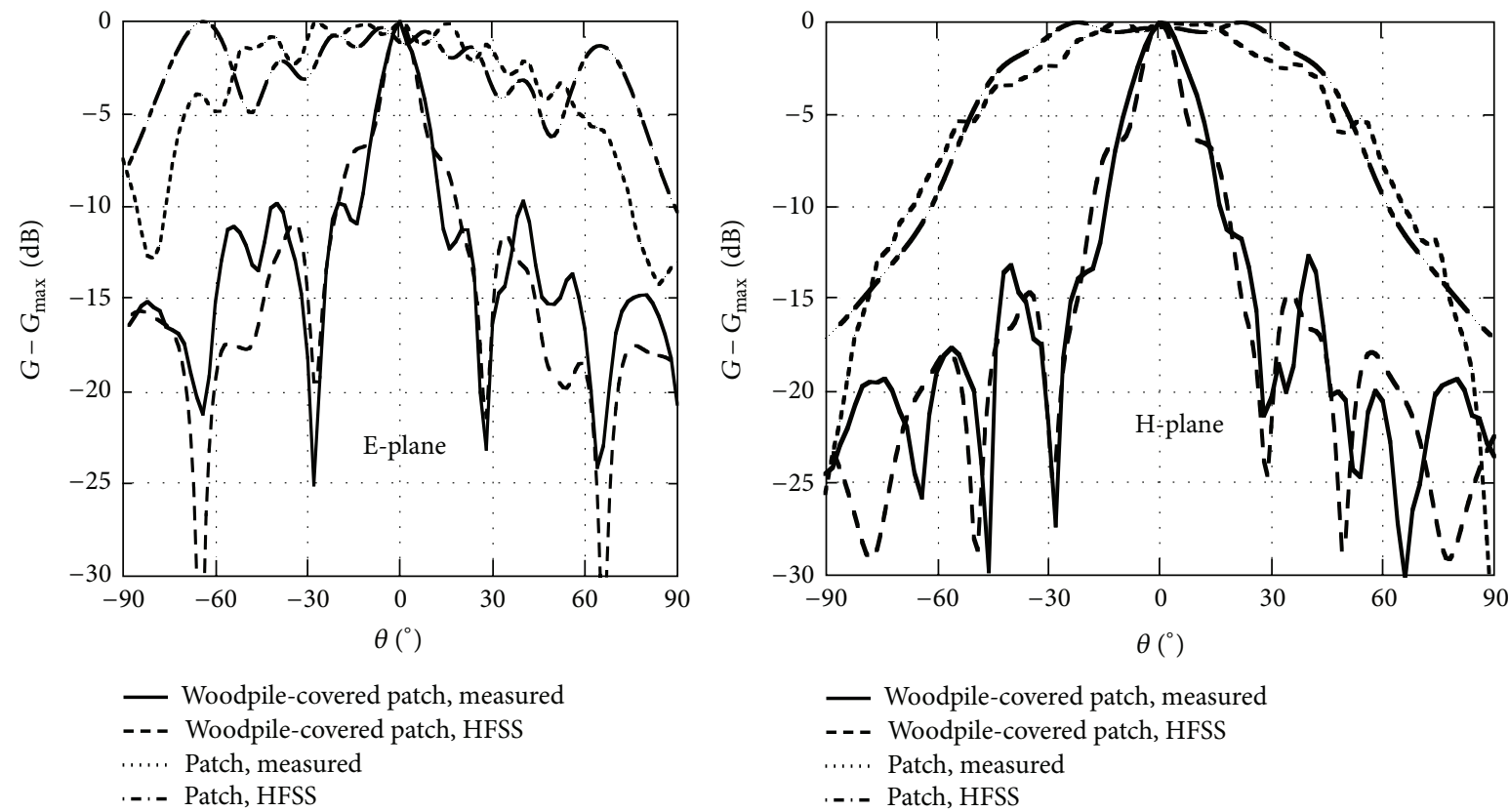

FIGURE 19: E- and H-plane radiation patterns $(\mathrm{dB})$ of the woodpile-covered patch antenna, normalized to its maximum value; $h / 2=45 \mathrm{~mm}$, and the bars nearest to the patch are parallel to the electric field. Experimental results for the patch and curves obtained by using HFSS are reported, for comparison.

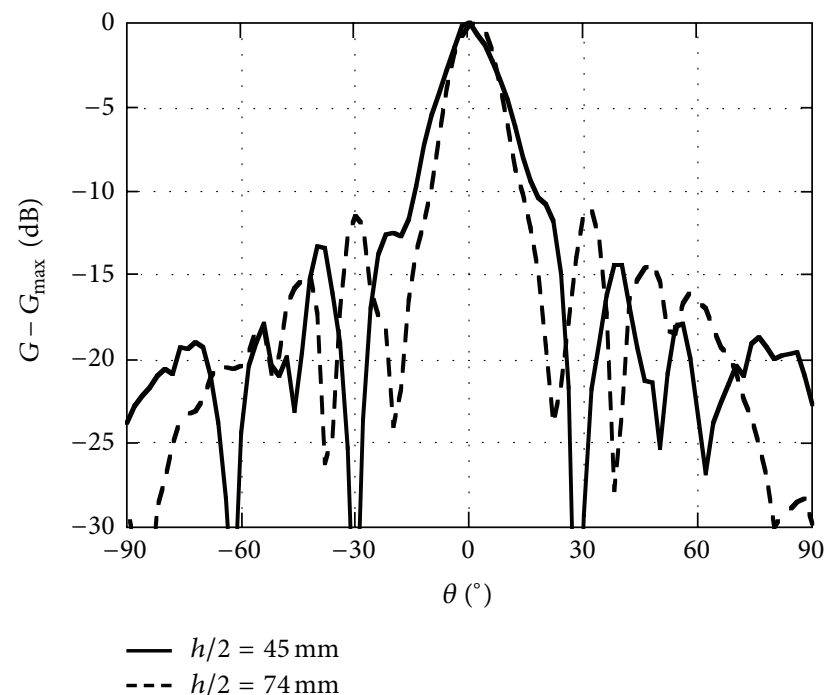

FIGURE 20: H-plane radiation pattern (dB) of the woodpile-covered patch antenna, normalized to its maximum value, when $h / 2=45$ and $74 \mathrm{~mm}$. The bars nearest to the patch are parallel to the electric field.

woodpile layers. The patch is the same as in the previous example. At $13.24 \mathrm{GHz}$, with the superstrate at a distance $h / 2=45 \mathrm{~mm}$, the gain enhancement due to the presence of the EBG is $18.28 \mathrm{~dB}$ and the return loss of the compound radiator is less than $-20 \mathrm{~dB}$. In Figure 25, the return loss of the

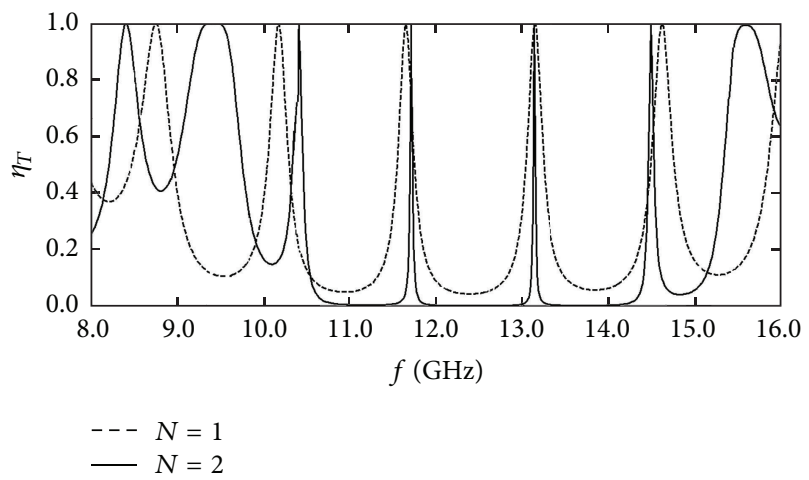

FIGURE 21: FMM results for a Fabry-Perot cavity with mirrors constituted by one ( $N=1$, dashed line) or two ( $N=2$, solid line) woodpile layers: transmission as a function of frequency.

EBG resonator antenna is plotted as a function of frequency and it is compared with the return loss of the patch alone; the matching of the patch is slightly affected by the introduction of the double-woodpile superstrate. In Figure 26, the broadside gain of the EBG resonator antenna, $G_{\mathrm{pw}}$, normalized to the broadside gain of the patch alone, $G_{p}$, in $\mathrm{dB}$, is plotted as a function of frequency; the peak of gain enhancement is higher and narrower than in Figure 23. Finally, in Figure 27 we report the E- and $\mathrm{H}$-plane radiation patterns for this antenna; the HPBW is $9^{\circ}$ in the E-plane, $9^{\circ}$ in the $\mathrm{H}$-plane; the SLL is $-16.99 \mathrm{~dB}$ in the E-plane, $-20.23 \mathrm{~dB}$ in the $\mathrm{H}$-plane. 


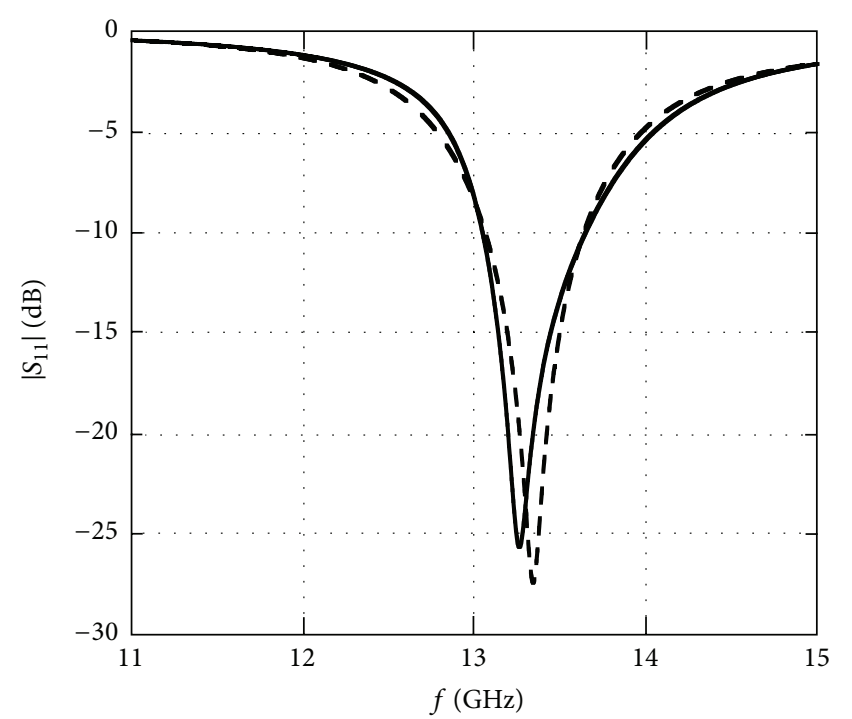

— Woodpile-covered patch, HFSS

- - - Patch, HFSS

FIGURE 22: Return loss of the woodpile-covered patch antenna resonating at $13.3 \mathrm{GHz}$ and of the patch alone, versus frequency; the distance between patch and woodpile is $h / 2=45 \mathrm{~mm}$.

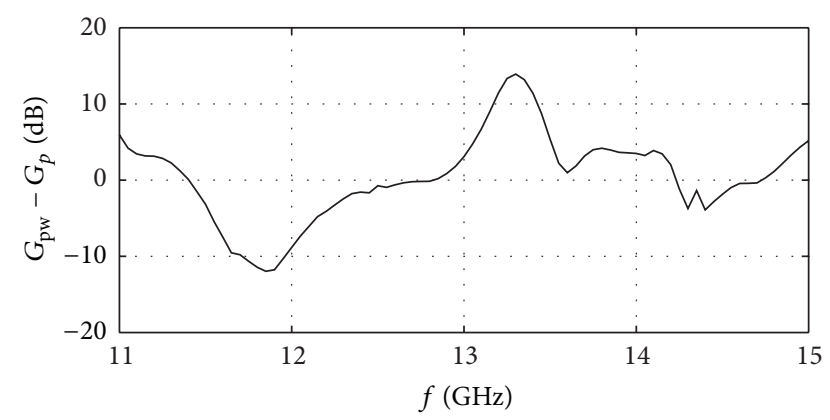

FIGURE 23: Broadside gain of the woodpile-covered patch antenna, $G_{\mathrm{pw}}$, normalized to the broadside gain of the patch alone, $G_{p}$, in $\mathrm{dB}$, versus frequency; the structure is the same as in Figure 22.

These results show that the introduction of a second woodpile layer further improves the gain enhancement.

\section{Conclusions}

The synthesis, realization and experimental characterization of a woodpile resonator have been presented. Its application for gain-enhancement of a microstrip patch antenna has been treated.

The Electromagnetic Band-Gap (EBG) material has been designed by using an in-house code implementing the Fourier Modal Method (FMM). It shows a stop-band centred on $12 \mathrm{GHz}$, extending over a large frequency interval and scarcely sensitive to variations of both incidence direction and field polarization.

Two woodpile samples have been fabricated, by means of alumina rods with square cross-section. The alignment of the rods was performed by means of a metallic structure, specifically designed, with equally spaced steel pins mounted on the four sides of an aluminum plate. A teflon/PVC support has been designed and realized, to hold the woodpile samples during the experimental investigations.

The transmission properties of the woodpile samples and of woodpile resonators have been measured. The cavity length and the orientation of the EBG mirrors have been varied. Several new and interesting experimental results have been reported. All the measurements have been performed in the $8-12 \mathrm{GHz}$ frequency range, in a shielded anechoic chamber, using a vector network analyzer. Comparisons have been performed with FMM, obtaining a good agreement.

We have verified that the EBG cavity resonates when its equivalent length is an integer multiple of half a wavelength. Moreover, we have found that the equivalent length of the cavity is highly dependent on the electromagnetic field polarization with respect to the woodpile orientation, covering the whole length of the air region plus the thickness of two or three periodic layers of both the mirrors, when the inner rods of the cavity are parallel or orthogonal to the electric field, respectively.

We have shown that the periodic arrangement of bars perpendicular to the electric field has a negligible effect on the transmission through the whole cavity. This suggests that adjacent layers of a woodpile might be independently designed, to interact with different linear sources having the electric field parallel to their rods or to modify and control the polarization of an elliptically polarized field.

The return loss, the gain, and the radiation pattern in the $\mathrm{E}$ - and $\mathrm{H}$-planes have subsequently been measured, for a rectangular patch antenna working at $10.3 \mathrm{GHz}$ and covered with the woodpile. Comparisons with the commercial software HFSS show a good agreement. We have observed that, when the equivalent distance between the patch an the EBG is roughly equal to an integer multiple of half a wavelength, the periodic superstrate does not significantly affect the antenna matching; the gain of the woodpilecovered antenna results to be about $10 \mathrm{~dB}$ greater than the gain of the patch alone. We have also found that if the distance between antenna and EBG is larger, the radiation pattern has a narrower main beam and a higher side-lobe level.

We have carried out a comparison between our EBG resonator antenna and two-dimensional uniform arrays of patches. With a 9-element array, covering an area smaller than the woodpile extension, a gain enhancement can be obtained slightly higher than the one achieved by our EBG resonator antenna. An array with a higher number of elements, occupying the whole woodpile area, shows a gain enhancement much stronger than the woodpile-covered patch. Therefore, when a directive beam is needed, a woodpile cover can be considered a good alternative to an array not in the sense that it gives a higher increase of the gain. One of the benefits of the EBG resonator antenna resides in the possibility of obtaining a high gain with a single 

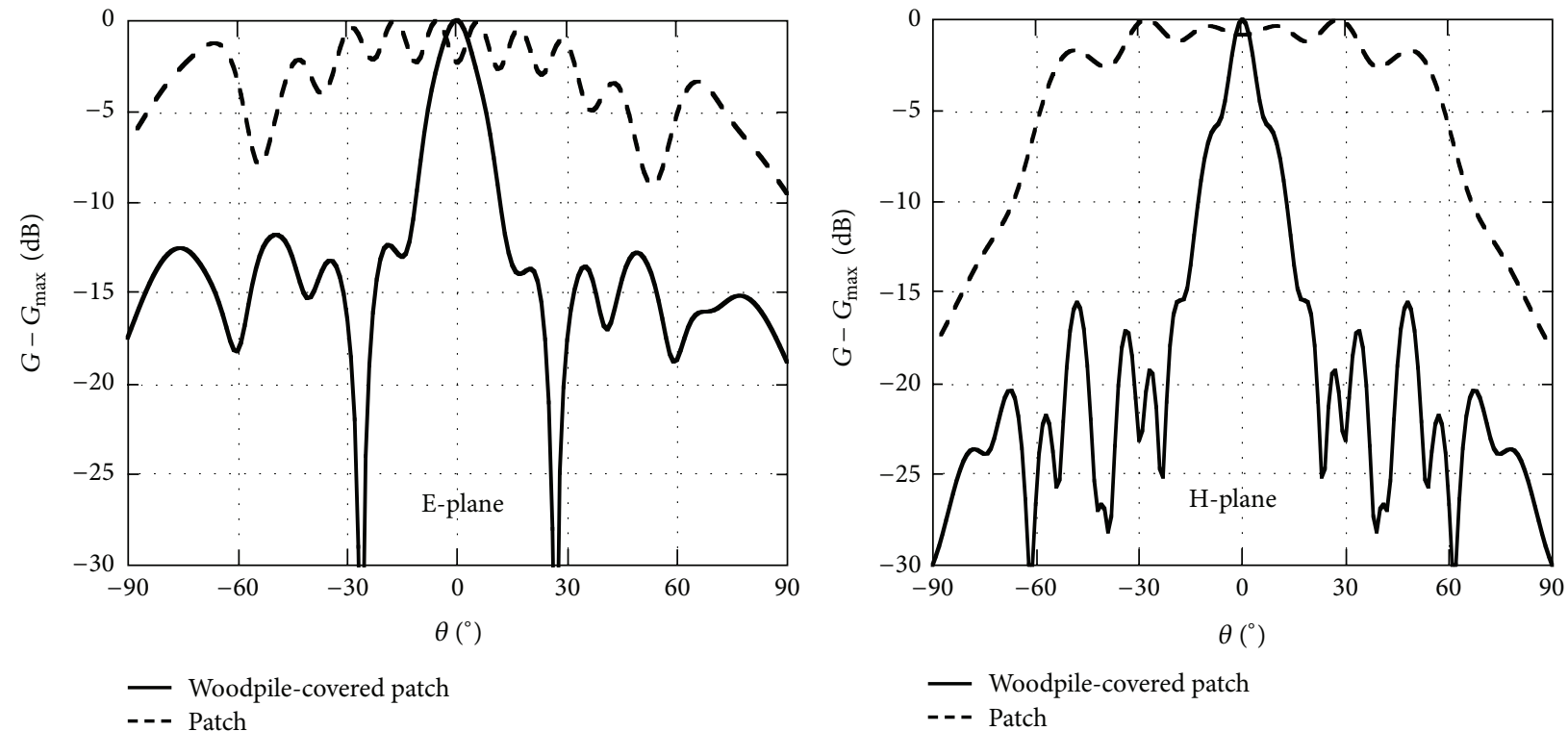

FIGURE 24: E- and H-plane radiation patterns for the woodpile-covered patch antenna, calculated at $13.3 \mathrm{GHz}$; the structure is the same as in Figure 22.

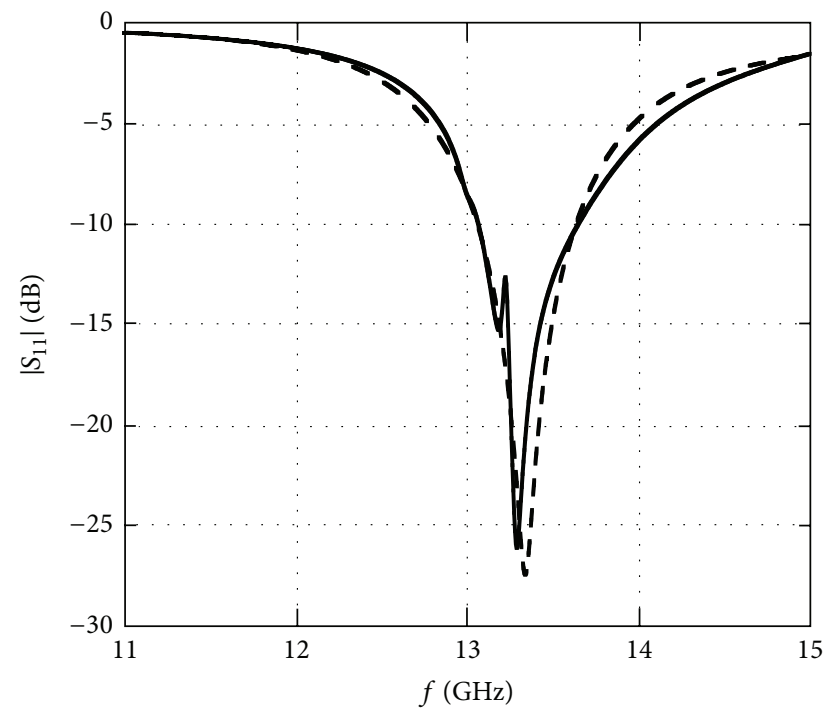

- Woodpile-covered patch, HFSS

- - - Patch, HFSS

FIGURE 25: Return loss of the patch antenna with a cover constituted by two woodpile layers, resonating at $13.24 \mathrm{GHz}$, compared with the return loss of the patch alone; the distance between patch and woodpile is $h / 2=45 \mathrm{~mm}$.

feeding device, without the complications of a multiplesource radiating system. Moreover, the matching of the antenna turns out to be slightly affected by the introduction of the periodic cover, while an array has a highly resonant matching.

By performing FMM and HFSS simulations, we have demonstrated that stronger gain enhancement can be

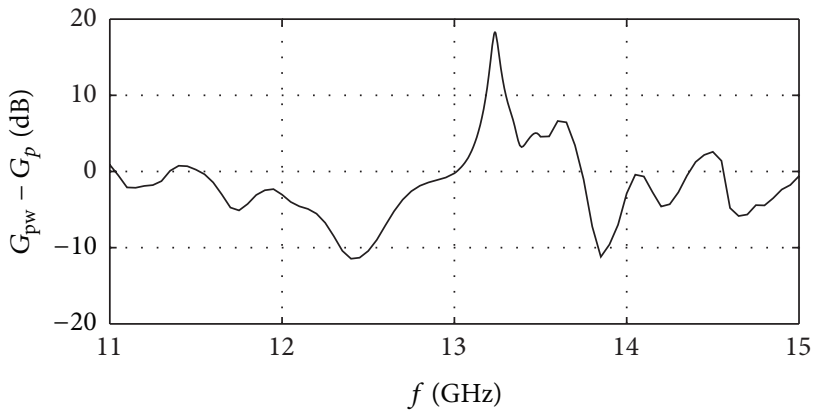

FIGURE 26: Broadside gain, $G_{\mathrm{pw}}$, normalized to the broadside gain of the patch alone, $G_{p}$, in $\mathrm{dB}$, versus frequency; the structure is the same as in Figure 25.

obtained working at higher frequencies and/or using a double-woodpile cover. The gain of a rectangular patch, covered with a double woodpile layer and resonating at 13.24 GHz, results to be more than $18 \mathrm{~dB}$ greater than the gain of the patch alone.

We think that an even higher gain might be reached by designing a modified woodpile structure, with some bars displaced and optimized to achieve a more symmetric and directive radiation pattern and a better matching. We plan to investigate this solution in the near future. We are also going to study the performances of multiple antennas, working simultaneously at different frequencies and/or with different polarizations and exploiting the same woodpile layer. Finally, as is known it is possible to obtain a highly directive antenna by embedding a source into an EBG working near its band-gap [36], thanks to the limited 


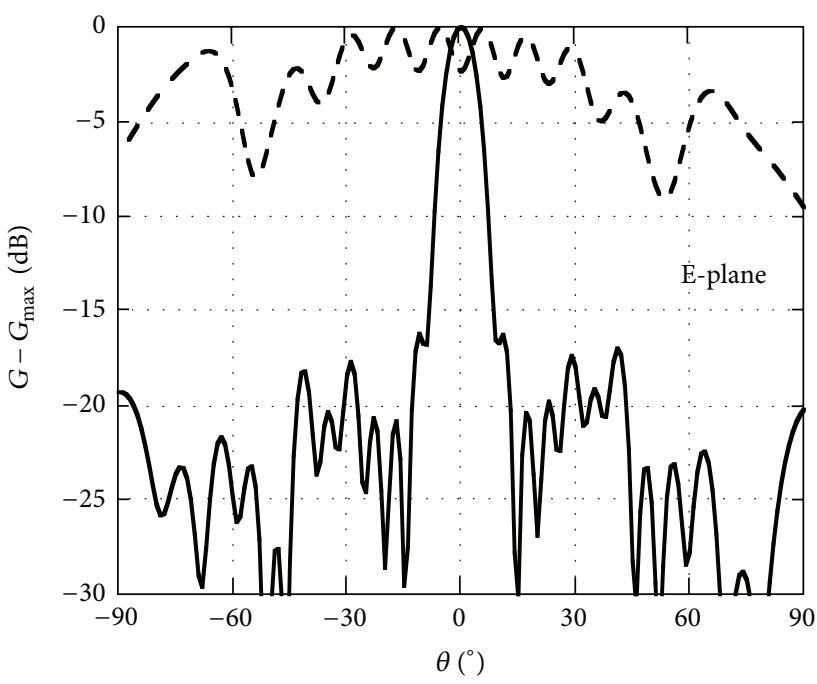

- Woodpile-covered patch - - - Patch

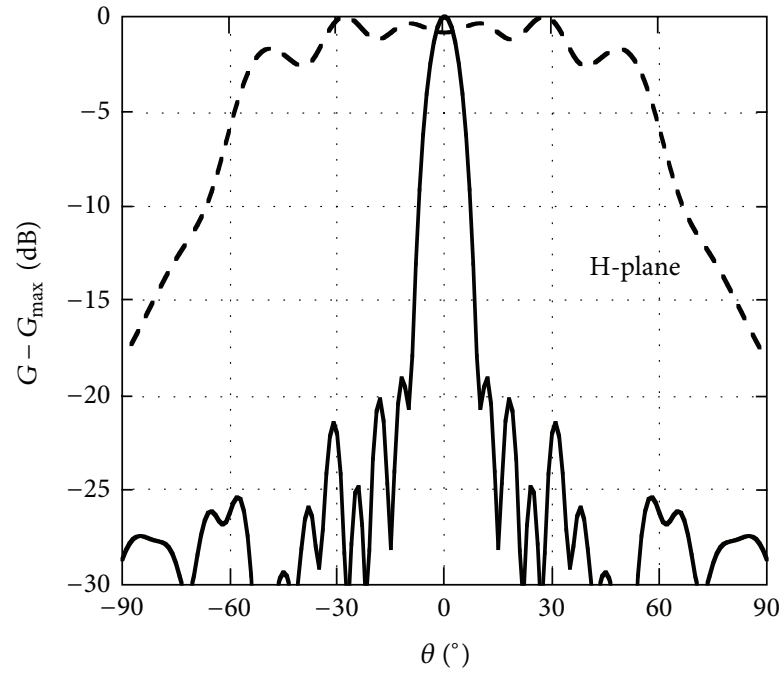

- Woodpile-covered patch - - Patch

FIGURE 27: E- and H-plane radiation patterns, calculated at $13.24 \mathrm{GHz}$; the structure is the same as in Figure 25.

angular propagation allowed within the crystal: we plan to compare this kind of EBG antenna with the EBG resonator antenna.

\section{Conflict of Interests}

The authors declare that there is no conflict of interests regarding the publication of this paper.

\section{References}

[1] J. D. Joannopoulos, S. G. Johnson, J. N. Winn, and R. D. Meade, Photonic Crystals: Molding the Flow of Light, Princeton University Press, Princeton, NJ, USA, 2nd edition, 2008.

[2] K. Yasumoto, Ed., Electromagnetic Theory and Applications for Photonic Crystals, CRC Press; Taylor \& Francis, New York, NY, USA, 2005.

[3] F. Yang and Y. Rahmat-Samii, Electromagnetic Band Gap Structures in Antenna Engineering, Cambridge University Press, New York, NY, USA, 2009.

[4] N. Engheta and R. W. Ziolkowski, Eds., Metamaterials: Physics and Engineering Explorations, Wiley-IEEE Press, Hoboken, NJ, USA, 2006

[5] K. M. Ho, C. T. Chan, C. M. Soukoulis, R. Biswas, and M. Sigalas, "Photonic band gaps in three dimensions: new layer-by-layer periodic structures," Solid State Communications, vol. 89, no. 5, pp. 413-416, 1994.

[6] H. S. Sözüer and J. P. Dowling, "Photonic band calculations for woodpile structures," Journal of Modern Optics, vol. 41, no. 2, pp. 231-239, 1994.

[7] S. Y. Lin, J. G. Fleming, D. L. Hetherington et al., "A threedimensional photonic crystal operating at infrared wavelengths," Nature, vol. 394, no. 6690, pp. 251-253, 1998.

[8] Y. Lee, X. Lu, Y. Hao, S. Yang, J. R. G. Evans, and C. G. Parini, "Low-profile directive millimeter-wave antennas using free-formed three-dimensional (3-D) electromagnetic bandgap structures," IEEE Transactions on Antennas and Propagation, vol. 57, no. 10, pp. 2893-2903, 2009.

[9] N. Tétreault, G. von Freymann, M. Deubel et al., "New route to three-dimensional photonic bandgap materials: silicon double inversion of polymer templates," Advanced Materials, vol. 18, no. 4, pp. 457-460, 2006.

[10] I. Staude, M. Thiel, S. Essig et al., "Fabrication and characterization of silicon woodpile photonic crystals with a complete bandgap at telecom wavelengths," Optics Letters, vol. 35, no. 7, pp. 1094-1096, 2010.

[11] A. Chutinan and S. Noda, "Highly confined waveguides and waveguide bends in three-dimensional photonic crystal," Applied Physics Letters, vol. 75, no. 24, pp. 3739-3741, 1999.

[12] A. Chutinan and S. Noda, "Design for waveguides in threedimensional photonic crystals," Japanese Journal of Applied Physics B, vol. 39, no. 4, pp. 2353-2356, 2000.

[13] M. Bayindir, E. Özbay, M. M. Sigalas, C. M. Soukoulis, R. Biswas, and K. M. Ho, "Guiding, bending and splitting of electromagnetic waves in highly confined photonic crystals," Physical Review B, vol. 63, no. 8, Article ID 081107, 4 pages, 2001.

[14] A. R. Weily, K. P. Esselle, T. S. Bird, and B. C. Sanders, "Experimental woodpile EBG waveguides, bends and power dividers at microwave frequencies," IEE Electronics Letters, vol. 42, no. 1, pp. 32-33, 2006.

[15] I. Ederra, R. Gonzalo, B. Martinez et al., "Modifications of the woodpile structure for the improvement of its performance as substrate for dipole antennas," IET Microwaves, Antennas and Propagation, vol. 1, no. 1, pp. 226-233, 2007.

[16] A. R. Weily, L. Horvath, K. P. Esselle, B. C. Sanders, and T. S. Bird, "A planar resonator antenna based on a woodpile EBG material," IEEE Transactions on Antennas and Propagation, vol. 53, no. 1, pp. 216-223, 2005.

[17] F. Frezza, L. Pajewski, and G. Schettini, "Numerical investigation on the filtering behavior of 2-D PBGs with multiple periodic defects," IEEE Transactions on Nanotechnology, vol. 4, no. 6, pp. 730-739, 2005. 
[18] A. Coves, S. Marini, B. Gimeno, and V. Boria, "Full-wave analysis of periodic dielectric frequency-selective surfaces under plane wave excitation," IEEE Transactions on Antennas \& Propagation, vol. 60, no. 6, pp. 2760-2769, 2012.

[19] E. Özbay and B. Temelkuran, "Reflection properties and defect formation in photonic crystals," Applied Physics Letters, vol. 69, no. 6, pp. 743-745, 1996.

[20] H. Němec, L. Duvillaret, F. Quemeneur, and P. Kužel, “Defect modes caused by twinning in one-dimensional photonic crystals," Journal of the Optical Society of America B, vol. 21, no. 3, pp. 548-553, 2004.

[21] H. Němec, P. Kužel, F. Garet, and L. Duvillaret, “Time-domain terahertz study of defect formation in one-dimensional photonic crystals," Applied Optics, vol. 43, no. 9, pp. 1965-1970, 2004.

[22] F. Frezza, L. Pajewski, and G. Schettini, "Periodic defects in 2D-PBG materials: full-wave analysis and design," IEEE Transactions on Nanotechnology, vol. 2, no. 3, pp. 126-134, 2003.

[23] H.-Y. D. Yang, N. G. Alexopoulos, and E. Yablonovitch, "Photonic band-gap materials for high-gain printed circuit antennas," IEEE Transactions on Antennas and Propagation, vol. 45, no. 1, pp. 185-187, 1997.

[24] Y. J. Lee, J. Yeo, R. Mittra, and W. S. Park, "Application of Electromagnetic BandGap (EBG) superstrates with controllable defects for a class of patch antennas as spatial angular filters," IEEE Transactions on Antennas \& Propagation, vol. 53, no. 1, pp. 224-235, 2005.

[25] M. Thevenot, J. Drouet, R. Chantalat, E. Arnaud, T. Monediere, and B. Jecko, "Improvements for the EBG resonator antenna technology," in Proceedings of the 2nd European Conference on Antennas and Propagation (EuCAP '07), pp. 526-531, Edinburgh, UK, November 2007.

[26] R. Biswas, E. Özbay, B. Temelkuran, M. Bayindir, M. M. Sigalas, and K.-M. Ho, "Exceptionally directional sources with photonic-bandgap crystals," Journal of the Optical Society of America B, vol. 18, no. 11, pp. 1684-1689, 2001.

[27] E. Özbay, B. Temelkuran, and M. Bayindir, "Microwave applications of photonic crystals," Progress in Electromagnetic Research, vol. 41, pp. 185-209, 2003.

[28] R. Sauleau, P. Coquet, T. Matsui, and J.-P. Daniel, "A new concept of focusing antennas using plane-parallel Fabry-Perot cavities with nonuniform mirrors," IEEE Transactions on Antennas and Propagation, vol. 51, no. 11, pp. 3171-3175, 2003.

[29] A. R. Weily, K. P. Esselle, B. C. Sanders, and T. S. Bird, "A woodpile EBG sectoral horn antenna," in Proceedings of the IEEE Antennas and Propagation Society International Symposium, vol. 4B, pp. 323-326, Washington, DC, USA, July 2005.

[30] A. R. Weily, K. P. Esselle, T. S. Bird, and B. C. Sanders, "Linear array of woodpile EBG sectoral horn antennas," IEEE Transactions on Antennas and Propagation, vol. 54, no. 8, pp. 2263-2274, 2006.

[31] F. Frezza, L. Pajewski, and G. Schettini, "Characterization and design of two-dimensional electromagnetic band-gap structures by use of a full-wave method for diffraction gratings," IEEE Transactions on Microwave Theory and Techniques, vol. 51, no. 3, pp. 941-951, 2003.

[32] F. Frezza, L. Pajewski, and G. Schettini, "Full-wave characterization of three-dimensional photonic band-gap structures," IEEE Transactions on Nanotechnology, vol. 5, no. 5, pp. 545-553, 2006.

[33] L. Pajewski and G. Schettini, "Three-dimensional electromagnetic band-gap structures: theory and applications," in Advanced Techniques for Microwave Systems, G. Schettini, Ed., chapter 4, pp. 59-84, Research Signpost, Trivandrum, India, 2012.

[34] F. Frezza, L. Pajewski, E. Piuzzi, C. Ponti, and G. Schettini, "Design and fabrication of a 3D-EBG superstrate for patch antennas," in Proceedings of the 39th European Microwave Conference (EuMC '09), pp. 1496-1499, Rome, Italy, SeptemberOctober 2009.

[35] F. Frezza, L. Pajewski, E. Piuzzi, C. Ponti, and G. Schettini, "Analysis and experimental characterization of an alumina woodpile-covered planar antenna," in Proceedings of the 40th European Microwave Conference (EuMC '10), pp. 200-203, Paris, France, September 2010.

[36] L. Pajewski, L. Rinaldi, and G. Schettini, "Enhancement of directivity using 2D-electromagnetic crystals near the bandgap edge: a full-wave approach," Progress in Electromagnetics Research, vol. 80, pp. 179-196, 2008. 

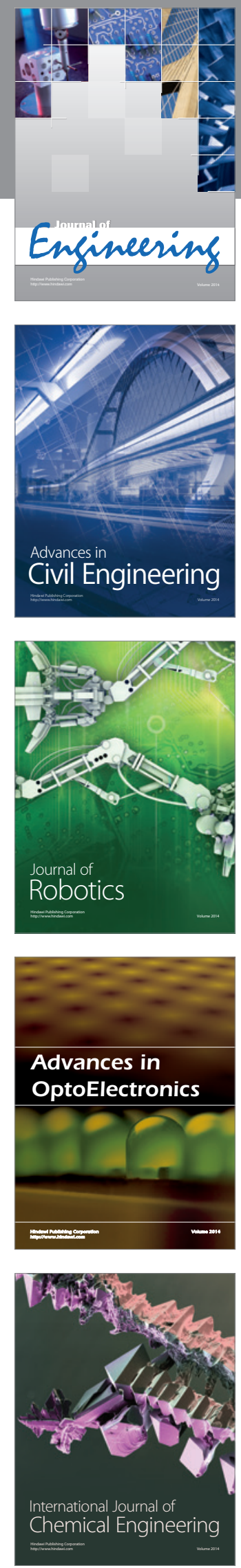

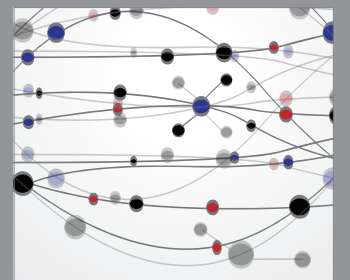

The Scientific World Journal
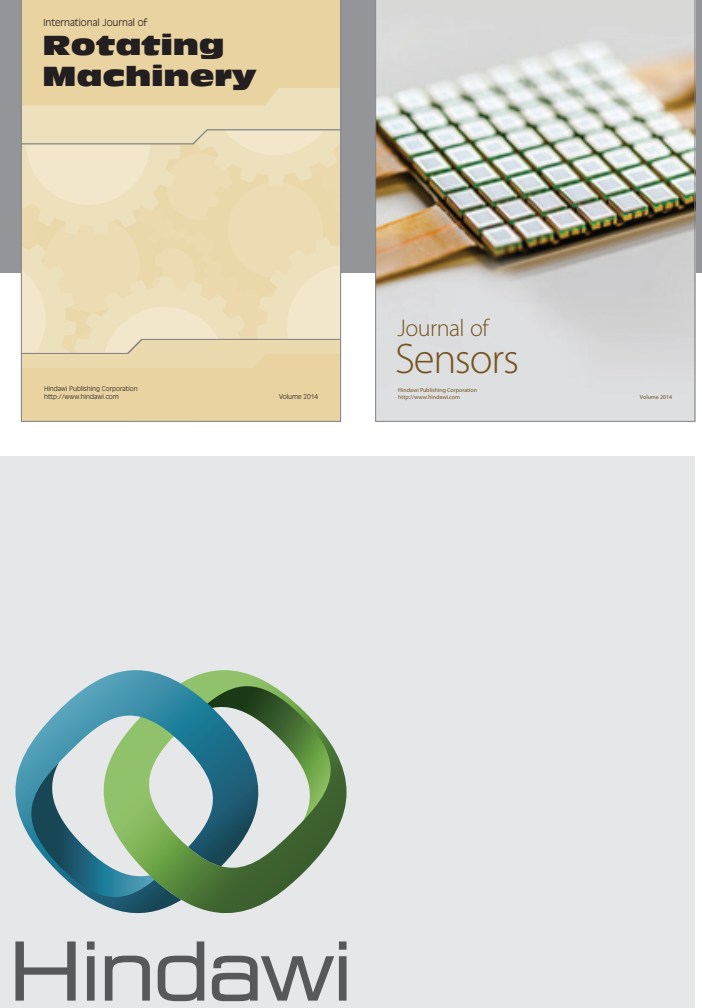

Submit your manuscripts at http://www.hindawi.com
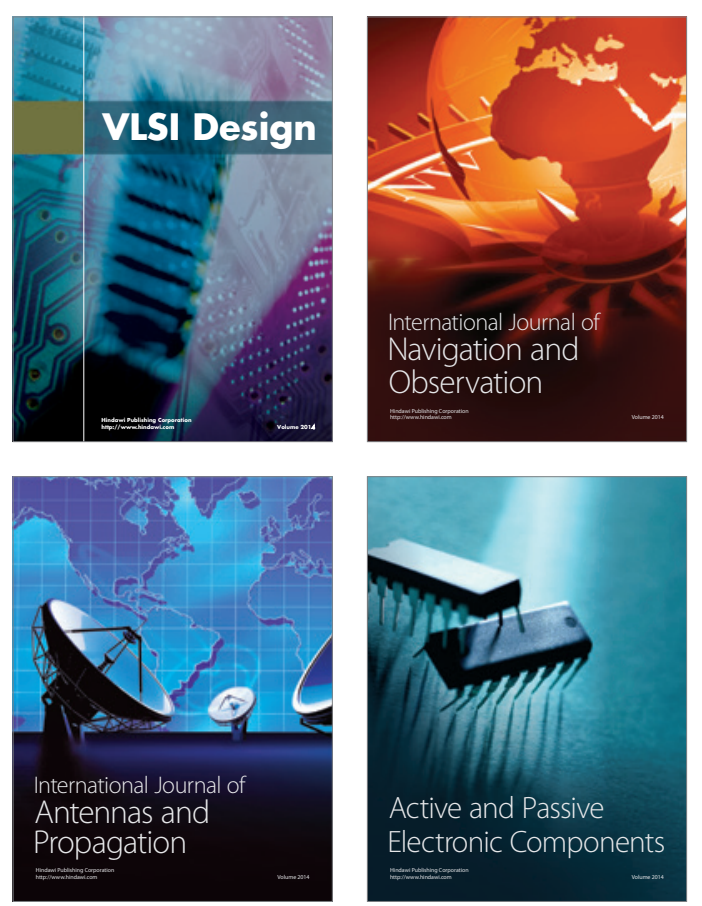
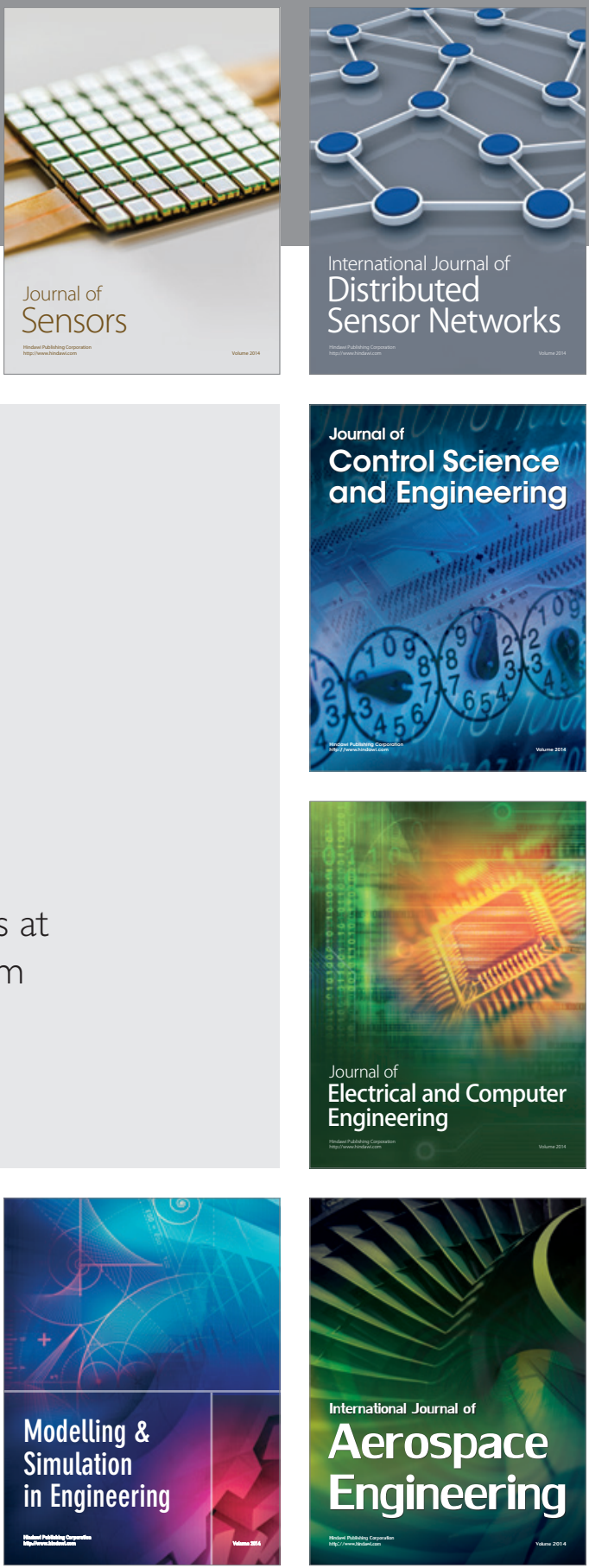

Journal of

Control Science

and Engineering
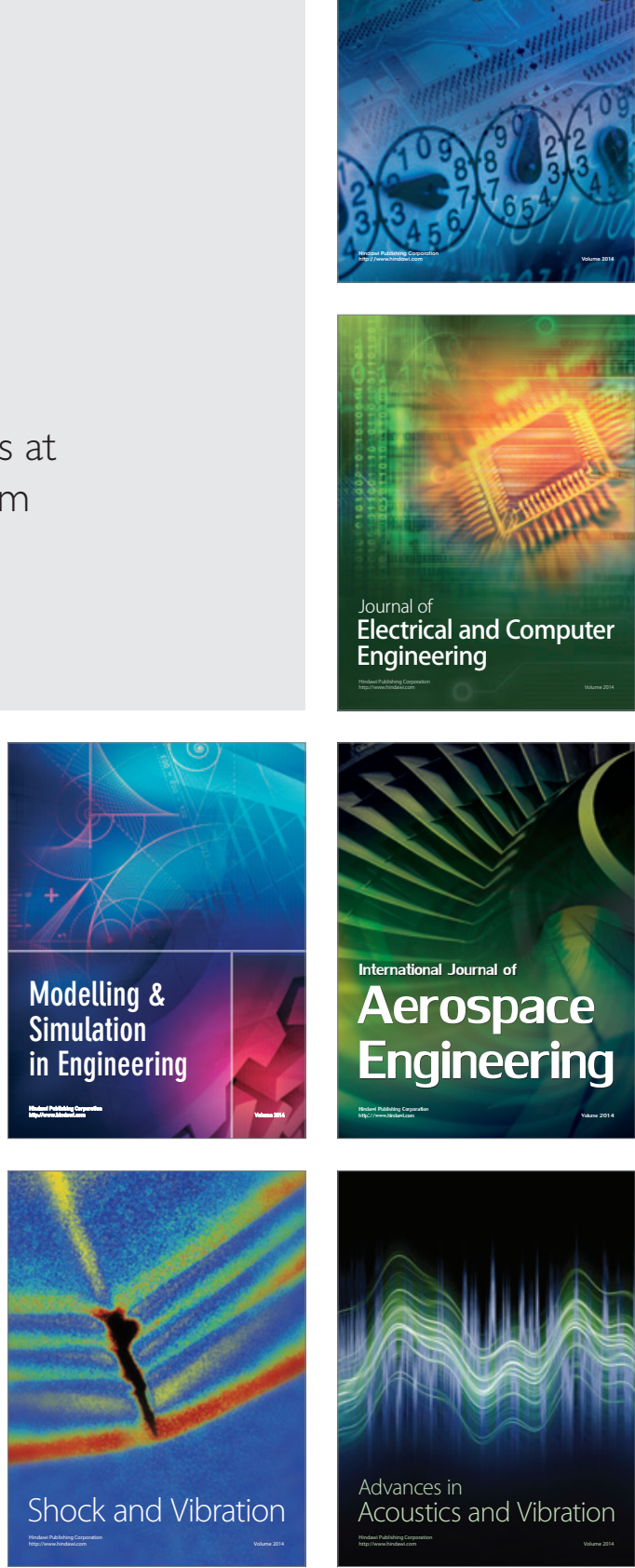